This item was submitted to Loughborough's Research Repository by the author.

Items in Figshare are protected by copyright, with all rights reserved, unless otherwise indicated.

\title{
Performance tradeoff of MVNOs in OFDMA-based virtualized wireless networks
}

PLEASE CITE THE PUBLISHED VERSION

https://doi.org/10.1109/TVT.2021.3124312

\section{PUBLISHER}

Institute of Electrical and Electronics Engineers

VERSION

AM (Accepted Manuscript)

\section{PUBLISHER STATEMENT}

(c) 2021 IEEE. Personal use of this material is permitted. Permission from IEEE must be obtained for all other uses, in any current or future media, including reprinting/republishing this material for advertising or promotional purposes, creating new collective works, for resale or redistribution to servers or lists, or reuse of any copyrighted component of this work in other works.

\section{LICENCE}

\section{All Rights Reserved}

\section{REPOSITORY RECORD}

Mili, Mohammad Robat, Shenhong Li, Fatemeh Mokhtari, Mahsa Derakhshani, Farid Ashtiani, and Tho LeNgoc. 2021. "Performance Tradeoff of Mvnos in Ofdma-based Virtualized Wireless Networks". Loughborough University. https://hdl.handle.net/2134/16910218.v1. 


\title{
Performance Tradeoff of MVNOs in OFDMA-based Virtualized Wireless Networks
}

\author{
Mohammad Robat Mili, Shenhong Li, Fatemeh Mokhtari, Mahsa Derakhshani, \\ Farid Ashtiani and Tho Le-Ngoc
}

\begin{abstract}
In this paper, we analyze the tradeoff between the profits gained by mobile virtual network operators (MVNOs) in an orthogonal frequency division multiple access (OFDMA)based virtualized wireless network (VWN). In this respect, MVNOs rent the network resources from a mobile network operator (MNO) to create virtual resources based on allocated rates and the cost due to allocated transmit powers in two different strategies: resource-based isolation strategy and ratebased isolation strategy. In resource-based isolation strategy, it is assumed that the whole bandwidth in each base station is divided equally between MVNOs whereas in rate-based isolation strategy, the whole bandwidth in each base station is allocated dynamically between MVNOs. We then formulate a multiobjective optimization problem (MOOP) to show the tradeoff between the maximum profit of each MVNO. To solve the formulated MOOP, we adopt the weighted Tchebycheff approach, which can provide the complete Pareto optimal region for nonconvex problems. Thus, by developing such formulation, we will be able to find the best profit gained by MVNOs. We also employ monotonic optimization to obtain the global optimal solutions of the non-convex problems by applying polyblock outer approximation algorithm. Our numerical results show the importance of the aforementioned tradeoffs and confirm that the proposed geometric programming (GP) based algorithm achieves excellent performance as compared to the monotonic method.
\end{abstract}

\section{INTRODUCTION}

In the current decade, due to the tremendous growth of wireless traffic and services, the mobile networks require a fundamental change to provide higher capacity and to exploit the available capacity more efficiently. Some recent studies have indicated a growing worldwide demand in network provisioning for data-dominated services, e.g., video and social network applications [1]. The concept of virtualized wireless networks (VWNs) has been proposed as a promising approach to increase the spectrum efficiency for next-generation wireless networks where virtual network operators can efficiently use

Copyright (c) 2021 IEEE. Personal use of this material is permitted. However, permission to use this material for any other purposes must be obtained from the IEEE by sending a request to pubs-permissions@ieee.org.

This work was supported by the Royal Academy of Engineering under the Leverhulme Trust Research Fellowship scheme under Grant DerakhshaniLTRF1920 $16 \backslash 67$.

Mohammad Robat Mili, Fatemeh Mokhtari and Farid Ashtiani are with the Department of Electrical Engineering, Sharif University of Technology, Tehran, Iran (email: mohammad.robatmili@ieee.org; fhmokhtari@gmail.com; ashtianimt@sharif.edu)

Shenhong Li is with the School of Information Science and Technology, Nantong University, Nantong, 226019, China (email: shenhongli@ntu.edu.cn)

Mahsa Derakhshani is with the Signal Processing and Networks Research Group, Wolfson School, Loughborough University, Leicestershire, LE11 3TU, United Kingdom (email: m.derakhshani@lboro.ac.uk).

Tho Le-Ngoc is with the Department of Electrical \& Computer Engineering, McGill University, Montreal, QC, Canada (email: tho.le-ngoc@mcgill.ca). the shared limited wireless resources [2], [3], e.g., infrastructure, radio spectrum and computation resources owned and managed by infrastructure providers (InPs) [4]. Driven by the ever-increasing market demand, mobile virtual network operators (MVNOs) [5] have rapidly popularized and achieved commercial success in recent years. In 2018, the global MVNO market revenue has reached approximately 60.5 billion U.S. dollars, and it is expected to grow to 102.932 billion U.S. dollars in 2023. In addition, the number of MVNOs worldwide has exceeded 1000 [6]. MVNO is a new type of wireless communication service provider which offers cheaper or more flexible data plans. An MVNO is a mobile carrier that provides mobile phone services, while it does not have its own licensed frequency allocation of radio spectrum, nor does it necessarily have the entire infrastructure required to provide mobile telephone services [7]. MVNOs can be classified into two types, full and light [8]. Full MVNOs depend only on their host MNO's radio access network and part of the Internet Protocol (IP) core network. Light MVNOs almost entirely depend on their host MNO's infrastructure, including the radio access network and IP core work, but they provide their own authentication servers. In [9], the authors proposed an efficient resource allocation framework by using the Generalized Kelly Mechanism (GSK). An InP first allocates its wireless resources among MVNOs based on auction mechanism, and then each MVNO efficiently shares the obtained wireless resources from the $\mathrm{InP}$ to its mobile users. In network virtualization environment [10], complicated physical network can be separated into several simple virtual networks which makes multi-service resource allocation problem simpler. The main goal of VWNs is the separation of services and physical resources. An efficient enabler for this scheme is the new entities, i.e., MVNOs [11][14]. To this end, MVNOs rent physical resources from InP in the form of slices and employ them as virtual resources to provide services for users [15]. As a result, MVNOs are able to customize the resources in order to provide bespoke services based on their qualities defined by service providers (SPs). Since the SPs need to pay to MVNOs for their QoS provisioning, MVNOs should efficiently allocate the available resources to enhance the SP performance. Furthermore, the capital expenditures and operating expenses by using VWNs can be significantly reduced [2], [11], [15], [16].

In most previous works on resource allocations in VWNs, a single objective optimization problem (SOOP) is solved to maximize the network sum rate [9], [17]-[22]. For example, adaptive resource allocation techniques have been considered in orthogonal frequency division multiple access (OFDMA)- 
based VWNs in order to maximize the network sum rate subject to constraints on the minimum required rate for each slice and individual base-station transmit power [17]-[19]. More specifically, a joint BS assignment, sub-carrier, and power allocation algorithm is formulated and solved in [17] to maximize the network sum rate, while satisfying the minimum required rate of each slice. The resource allocation for wireless virtualized networks is studied in [18] when both the backhaul capacity of the infrastructure provider and the users' quality-of-service requirements are guaranteed. The resource allocation problem is formulated as an NP-hard optimization problem that jointly optimizes power and slice allocations. Moreover, in [22], a coalitional game-based algorithm is proposed to maximize the system-wide utility and minimize the total power consumption through jointly optimizing the user association and power allocation for NOMA-enabled small-cell networks. In [19], the joint resource provisioning and admission control are formulated aiming to maximize the total rate of wireless virtualized networks while taking into account the minimum required rate and resources of rate-based and resource-based slices. The spectrum sharing problem in a heterogeneous wireless network is considered in [23] where small-cell base stations are optimally matched with SPs.

Moreover, pricing approach is considered as a popular method in wireless network virtualization. The purpose of the pricing approach is that the MNO (seller) shares the resources with others based on the competition of the resource price which is the bidding price from the MVNOs (buyers). Based on the bidding price from the buyers, the winner is selected and the optimal price is decided considering the revenue of the buyer and seller. In [24], the dynamic pricing that the InP applies to MVNOs is modeled as a two-stage Stackelberg game. A multi-stage game for modeling the MNO-MVNO interaction is presented in [25] where the MNO investment, the MVNO's decision on the leasing from the MNO and the retail pricing are successively focused on at each stage. In [26], the authors used backward induction to characterize a dynamic game for competitive spectrum acquisition and pricing strategies between two MVNOs. The authors in [27], studied how the MVNOs should make pricing decisions when others' inventory information is known or unknown. For the known case, they proposed an optimal pricing scheme for maximizing the revenue of each other. For the unknown case, a distributed coalition formation algorithm is developed to maximize each MVNO's revenue. In [28], the authors studied the complex interactions among the host MNO, MVNOs and end user under Cournot and Stacklberg games. In [29], the authors investigated the resource allocation problem in a virtualized-cloud radio access network (V-CRAN) as a two level non-cooperative pricing problem. In [30], the authors considered three-layer game theoretic model for maximization utilities of wireless resource providers (WRPs), MVNOs and end-users, and analyzed the uniqueness and existence of equilibrium point. Such pricing approaches are effective in optimizing the tradeoff between MVNOs for networks where distributed operation of different entities are plausible and/or required. In a network with centralized operation, multiobjective optimization (MOOP) can properly model and tackle the tradeoff between the profits of individual MVNOs.

Since the MVNOs usually consider different numbers of slices and frequency channels, the most benefit of MVNOs can be highly motivated by optimizing their profits as a performance metric. Therefore, allocating resources to the users of different MVNOs is conflicting. In other words, in the first glance, each MVNO does the best to optimize its profits, however, considering limited resources, the optimization on these profits would be considered as an MOOP [31].

The framework of MOOP has been applied to study the tradeoff between conflicting objectives in wireless systems [32]-[40]. For example, in [33], using the MOOP concept, the achievable sum rate and total power consumption were optimized while considering the downlink transmission of cloud-radio access networks (C-RANs) with limited fronthaul capacity. In [36], the authors presented a MOOP framework for energy efficiency and spectral efficiency maximization when radio resources are shared among multiple operators in a network. Also, in [37], to study a tradeoff between the energy efficiency and spectral efficiency in multicell heterogeneous networks, a MOOP is formulated in which both energy efficiency and spectral efficiency of the network are maximized while satisfying the rate requirement of users. A multi-objective power efficient resource allocation scheme for achieving balance between downlink and uplink transmit power minimization was proposed in [39]. Multi-objective resource allocation problem was formulated in [41] to investigate joint bandwidth and power allocation in multi-RAT HetNets to improve the energy efficiency. Moreover, in [42], the tradeoff among the downlink rates of SPs is improved while using non-orthogonal multiple access (NOMA) technique.

Different from previous papers in VWNs that studied the problem of resource allocations as a SOOP, in this paper, we exploit the MOOP framework in order to find a tradeoffs between the profits gained by MVNOs while guaranteeing different QoS corresponding to each MVNO. It is worth noting that because of nononvex nature of optimization on the considered performance metrics, the weighted sum of the metrics as a SOOP formulation is not able to provide the whole Pareto optimal region [43]. The main contributions of this paper can be summarized as follows:

- We first consider two different isolation strategies: resource-based and rate-based. In the resource-based isolation strategy, it is assumed that the whole bandwidth in each base station is divided equally between MVNOs whereas in the rate-based isolation strategy, the whole bandwidth in each base station is allocated dynamically between MVNOs.

- Optimization problems with multiple objectives appear naturally in many engineering fields to model tradeoffs between, for example, application performance, operational expenses, logistics, and environmental impacts. To analyze and obtain insights on such problems without imposing any additional structure it is common to formulate them as multi-objective optimization problems. A multi-objective optimization problem involves two or more optimization goals that can be conflicting, i.e., improvement to one objective comes at the expense of 
another objective. Since the MVNOs usually consider different numbers of slices and frequency channels, the most benefit of MVNOs can be highly motivated by optimizing their profits as a performance metric. Therefore, allocating resources to users of different MVNOs can be conflicting. In other words, in the first glance, each MVNO does the best to optimize its profits, however, considering limited resources, the optimization on these profits would be considered as a MOOP. We formulate an MOOP framework that jointly maximizes the profit of MVNOs to investigate a tradeoff between these profits for MVNOs in downlink OFDMA system for resourcebased and rate-based isolation strategies. The system is constrained by a maximum total transmit power for each base station.

- Towards solving the formulated MOOP, we first apply a weighted Tchebycheff method [31], [43] which is able to efficiently find the Pareto optimal solution while employing a much lower complexity in comparison with other methods, e.g., the weighted product method, epsilonmethod, and exponentially weighted criterion, despite the nonconvexity of the considered problem. Subsequently, we propose an iterative approach that alternatively assigns subchannels and allocates power to users at each MVNO. In this respect, for subchannel assignment, we relax the binary constraint of subchannel assignment indicator into continuous ones. For power allocation, the difference of convex (D.C.) programming is applied. The convergence of the proposed algorithm is also demonstrated.

- To find the global optimal solutions of SOOP, the SOOP is converted into the form of monotonic optimization which will reduce the difficulty. A majority of nonconvex problems for optimization of communication and networking systems exhibit monotonicity or hidden monotonicity structures. Thus, the optimal solutions can be found at the upper boundary of the feasible set by applying the polyblock outer approximation algorithm. In this paper, we also develop a non-convex optimization framework based on monotonic optimization by outer polyblock approximation. For doing so, we convert the MOOP into SOOP applying the weighted Tchebycheff method. Then, the SOOPs are represented in terms of differences of monotonic increasing functions. Next, the problems are reformulated as the maximization of increasing functions over normal constraint set.

The rest of this paper is organized as follows. Section II describes the system model of an OFDMA-based VWN. In Section III and Section IV, we formulate an MOOP between the profit of MVNOs and develop an algorithm to solve it by using weighted Tchebycheff method. The subchannel assignment and power allocation are separately investigated. In Section V, we also apply the monotonic optimization method to obtain the global optimal solutions. Numerical results are presented in Section VI, followed by concluding remarks in Section VII.

\section{SYSTEM MODEL}

We consider an OFDMA-based virtualized cellular network consisting of a set of $C$ cells (denoted by $\mathcal{C}=\{1,2, \ldots, C\}$ )

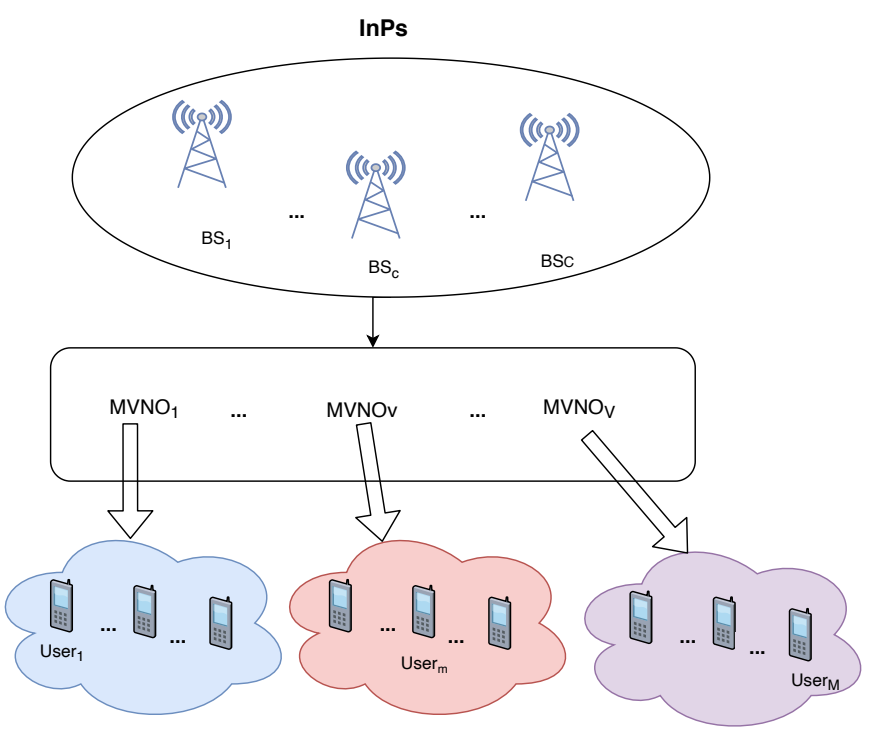

Fig. 1. Hierarchial model in VWNs

owned by InPs. As shown in Fig. 1, in this model, a set of $V$ MVNOs (denoted by $\mathcal{V}=\{1,2, \ldots, V\}$ ) rent the network resources from the InPs to serve the users in downlink. A set of all users is denoted by $\mathcal{M}=\{1,2, \ldots, M\}$.

Each user is exclusively subscribed to an MVNO and $\mathcal{I}_{v}$ denotes the set of users subscribed to MVNO $v \in \mathcal{V}$ such that $\bigcup_{\forall v \in \mathcal{V}} \mathcal{I}_{v}=\mathcal{M}$ and $\bigcap_{\forall v \in \mathcal{V}} \mathcal{I}_{v}=\emptyset$. The bandwidth of $B \mathrm{~Hz}$ is divided into $N$ sub-channels such that each subchannel has a bandwidth of $B_{c}=B / N \mathrm{~Hz}$. The set of all sub-channels is denoted by $\mathcal{N}$. The sub-channels are modeled as block flat-fading channels, where the channel gains remain constant during each time slot, but may vary independently from a time slot to another. Moreover, it is assumed that perfect channel state information of users is available. $h_{m}^{n, c}(t)$ denotes the instantaneous channel power gains from the BS of cell $c$ to user $m$ on sub-channel $n$ at time slot $t$.

Each user is associated with one cell and each sub-channel at each cell is allocated exclusively to one user due to the OFDMA restriction. The parameter $\psi_{m}^{n, c}$ represents subchannel assignment and cell association variable for user $m$. In particular, $\psi_{m}^{n, c}=1$ if sub-channel $n$ of cell $c$ is allocated to user $m$ and $\psi_{m}^{n, c}=0$ otherwise. Moreover, the power allocated for transmission for MVNO $v$ on sub-channel $n$ in cell $c$ at time slot $t$ is denoted by $p_{v}^{n, c}(t)$. The noise power spectrum density is given by $N_{0}$.

In this work, to manage isolation between different MVNOs, we consider two different strategies, i.e., resource-based and rate-based isolation. In the resource-based isolation strategy, an exclusive fixed bandwidth is allocated to each MVNO in each cell. Such strategy will allow us to allocate a predefined amount of sub-channels to each MVNO in each cell and avoid inter MVNO interference. Although such strategy helps to achieve resource-based isolation, its rigidity could limit the achievable performance. In contrast, we study rate-based isolation strategy that allows dynamic allocation of resources among MVNOs, while guaranteeing minimum rate require- 
ments per each MVNO.

In the following, we introduce each of these strategies in detail. In particular, we present the instantaneous received signalto-interference-plus-noise ratio (SINR) and the corresponding network utility for MVNOs.

\section{A. Resource-based Isolation Strategy}

In the resource-based isolation strategy, depending on the service level agreement, the entire bandwidth in each cell is divided between different MVNOs in a mutually exclusive manner. In particular, it is assumed that a fixed set of subchannels in each cell denoted by $\mathcal{N}_{v} \subset \mathcal{N}$ is allocated to MVNO $v$. With this strategy, the channel assignment and cell association variables should satisfy the following constraint.

$$
\sum_{\forall m \in \mathcal{I}_{v}} \sum_{\forall n \in \mathcal{N} \backslash \mathcal{N}_{v}} \sum_{\forall c \in \mathcal{C}} \psi_{m}^{n, c}=0, \forall v \in \mathcal{V},
$$

where $\mathcal{N} \backslash \mathcal{N}_{v}$ is the absolute complement of $\mathcal{N}_{v}$. Since the sub-channel subsets corresponding to different MVNOs (i.e., $\mathcal{N}_{v}$ 's) are mutually exclusive, no interference will be imposed to a user of an MVNO by other MVNOs.

In this case, the received signal-to-interference-plus-noise ratio (SINR) at user $m \in \mathcal{I}_{v}$ of MVNO $v$ on sub-channel $n \in \mathcal{N}_{v}$ in cell $c$ is given by ${ }^{1}$

$$
\mathrm{SINRS}_{m, v}^{n, c}=\frac{p_{v}^{n, c} h_{m}^{n, c}}{N_{0}+\sum_{\forall c^{\prime} \neq c} p_{v}^{n, c^{\prime}} h_{m}^{n, c^{\prime}}}, \forall m \in \mathcal{I}_{v}, \forall n \in \mathcal{N}_{v}
$$

where $h_{m}^{n, c^{\prime}}$ is the instantaneous channel power gain between the BS at cell $c^{\prime}$ and user $m$ on sub-channel $n$. Thus, $\sum_{\forall c^{\prime} \neq c} p_{v}^{n, c^{\prime}} h_{m}^{n, c^{\prime}}$ is the total interference experienced by user $m$ of MVNO $v$ in cell $c$ on sub-channel $n$ from other cells. We define the network utility of MVNO $v$ as the difference between the network revenue of MVNO $v$ acquired from providing resources to users and the cost incurred due to leasing the transmit power and bandwidth from base stations to serve its users as

$$
U_{v}^{\mathrm{S}}\left(\mathbf{p}_{v}, \boldsymbol{\psi}\right)=\kappa_{\mathrm{R}} R_{v}^{\mathrm{S}}\left(\mathbf{p}_{v}, \boldsymbol{\psi}\right)-\kappa_{\mathrm{P}} \Pi_{v}^{\mathrm{S}}\left(\mathbf{p}_{v}, \boldsymbol{\psi}\right)-\kappa_{\mathrm{B}} B_{v}^{\mathrm{S}}(\boldsymbol{\psi}),
$$

where

$$
R_{v}^{\mathrm{S}}\left(\mathbf{p}_{v}, \boldsymbol{\psi}\right)=\sum_{\forall m \in \mathcal{I}_{v}} \sum_{\forall n \in \mathcal{N}_{v}} \sum_{\forall c \in \mathcal{C}} \psi_{m}^{n, c} \ln \left(1+\operatorname{SINR} \mathcal{S}_{m, v}^{n, c}\right),
$$

is the achievable rates of all users subscribed to MVNO $v$,

$$
\Pi_{v}^{\mathrm{S}}\left(\mathbf{p}_{v}, \boldsymbol{\psi}\right)=\sum_{\forall m \in \mathcal{I}_{v}} \sum_{\forall n \in \mathcal{N}_{v}} \sum_{\forall c \in \mathcal{C}} \psi_{m}^{n, c} p_{v}^{n, c},
$$

is the total transmit power allocated to MVNO $v$ in all base stations, and

$$
B_{v}^{\mathrm{S}}(\boldsymbol{\psi})=\sum_{\forall m \in \mathcal{I}_{v}} \sum_{\forall n \in \mathcal{N}_{v}} \sum_{\forall c \in \mathcal{C}} \psi_{m}^{n, c},
$$

is the total bandwidth allocated to MVNO $v$. In (3), $\mathbf{p}_{v}$ represents the matrix of power allocated to MVNO $v$ in each sub-channel and each cell. $\kappa_{P}$ and $\kappa_{B}$ are the prices charged to the MVNOs for using the power and bandwidth, respectively. Moreover, $\kappa_{\mathrm{R}}$ is the revenue received per unit of data rate.

\footnotetext{
${ }^{1}$ Hereafter, the time index $t$ is removed for simplification of the notations.
}

\section{B. Rate-based Isolation Strategy}

With the rate-based isolation strategy, the whole bandwidth in each cell is dynamically distributed between MVNOs such that the frequency sub-channels allocated to a MVNO in one cell are not necessarily the same as the ones allocated to that MVNO in other cells. With such strategy, the SINR at user $m \in \mathcal{I}_{v}$ of MVNO $v$ on sub-channel $n$ in cell $c$ is given by

$$
\operatorname{SINR} \mathcal{T}_{m, v}^{n, c}=\frac{p_{v}^{n, c} h_{m}^{n, c}}{N_{0}+\sum_{\forall c^{\prime} \neq c} \sum_{\forall v^{\prime} \in \mathcal{V}} p_{v^{\prime}}^{n, c^{\prime}} h_{m}^{n, c^{\prime}}},
$$

where $h_{m}^{n, c^{\prime}}$ is the instantaneous channel power gain from the BS in cell $c^{\prime}$ to user $m$ on sub-channel $n$. Thus, $\sum_{\forall c^{\prime} \neq c} \sum_{\forall v^{\prime} \in \mathcal{V}} p_{v^{\prime}}^{n, c^{\prime}} h_{m}^{n, c^{\prime}}$ is the total interference from other cells and other MVNOs. Hence, the total achievable rates by users of MVNO $v$ is

$$
R_{v}^{\mathrm{T}}(\mathbf{p}, \boldsymbol{\psi})=\sum_{\forall m \in \mathcal{I}_{v}} \sum_{\forall n \in \mathcal{N}} \sum_{\forall c \in \mathcal{C}} \psi_{m}^{n, c} \ln \left(1+\operatorname{SINR} \mathcal{T}_{m, v}^{n, c}\right) .
$$

In this case, instead of exclusively assigning a certain amount of sub-channels to a MVNO, a minimum rate requirement is guaranteed as

$$
R_{v}^{\mathrm{T}} \geq R_{\min , v}, \forall v \in \mathcal{V}
$$

Similar to the resource-base isolation strategy, the network utility of MVNO $v$ is defined as

$$
U_{v}^{\mathrm{T}}(\mathbf{p}, \boldsymbol{\psi})=\kappa_{\mathrm{R}} R_{v}^{\mathrm{T}}(\mathbf{p}, \boldsymbol{\psi})-\kappa_{\mathrm{P}} \Pi_{v}^{\mathrm{T}}\left(\mathbf{p}_{v}, \boldsymbol{\psi}\right)-\kappa_{\mathrm{B}} B_{v}^{\mathrm{T}}(\boldsymbol{\psi}),
$$

where

$$
\Pi_{v}^{\mathrm{T}}\left(\mathbf{p}_{v}, \boldsymbol{\psi}\right)=\sum_{\forall m \in \mathcal{I}_{v}} \sum_{\forall n \in \mathcal{N}} \sum_{\forall c \in \mathcal{C}} \psi_{m}^{n, c} p_{v}^{n, c},
$$

is the total power allocated to MVNO $v$, and

$$
B_{v}^{\mathrm{T}}(\boldsymbol{\psi})=\sum_{\forall m \in \mathcal{I}_{v}} \sum_{\forall n \in \mathcal{N}} \sum_{\forall c \in \mathcal{C}} \psi_{m}^{n, c},
$$

is the total bandwidth assigned to MVNO $v$ in all base stations.

From the perspective of network service providers, how to maximize the network utility is always one of the major concerns. In this paper, we make use of utility function method for illustrating the business model of both InPs and MVNOs. The utility function of MVNO is defined as the difference between the network revenue of MVNO $v$ acquired from providing resources to users and the cost incurred due to leasing the transmit power and bandwidth from base stations to serve its users in (3). Here if the MVNO increases the power and bandwith allocated to its UEs, the data rate increases, and so does the revenue; however, the MNVO can suffer from higher cost due to the increased resource purchased from the InP. The difference between (2) and (7) is that the interference of (2) comes from the other cells, and the other interference in (7) comes from both other cells and other MVNOs.

In the following section, to analyze the tradeoff between maximum utilities of different MVNOs, we formulate the joint sub-channel assignment, cell association, and power control optimization problem as MOOP. 


\section{MOOP FORMULATION}

In this section, we formulate the MOOPs to assess the tradeoff between the profit of MVNOs for both resource-based and rate-based isolation strategies. The aim of the proposed MOOPs is to jointly provide the maximum profit for each MVNO under fading environment.

\section{A. Resource-based Isolation Strategy}

The joint constrained optimization for the resource-based isolation strategy can be formulated as

$$
\begin{aligned}
& \max _{\{\mathbf{p}, \boldsymbol{\psi}\}}\left\{U_{1}^{\mathrm{S}}, U_{2}^{\mathrm{S}}, \ldots, U_{v}^{\mathrm{S}}, \ldots, U_{V}^{\mathrm{S}}\right\} \\
& \text { s.t. } \\
& \sum_{\forall v \in \mathcal{V}} \sum_{\forall n \in \mathcal{N}_{v}} \sum_{\forall m \in \mathcal{I}_{v}} \psi_{m}^{n, c} p_{v}^{n, c} \leq P_{\text {max }}^{c}, \forall c \in \mathcal{C}, \\
& \sum_{\forall m \in \mathcal{M}} \psi_{m}^{n, c} \leq 1, \forall c \in \mathcal{C}, \forall n \in \mathcal{N}, \\
& \quad\left(\sum_{\forall n \in \mathcal{N}} \psi_{m}^{n, c}\right)\left(\sum_{\forall n \in \mathcal{N}} \sum_{\forall c^{\prime} \neq c} \psi_{m}^{n, c^{\prime}}\right)=0, \forall c \in \mathcal{C}, \forall m \in \mathcal{M},
\end{aligned}
$$$$
\psi_{m}^{n, c}=0, \forall m \in \mathcal{I}_{v} \text { and } n \in \mathcal{N} \backslash \mathcal{N}_{v}, \forall v \in \mathcal{V}, \forall c \in \mathcal{C},
$$$$
\psi_{m}^{n, c} \in\{0,1\} \quad \forall m \in \mathcal{M}, \forall n \in \mathcal{N}, \forall c \in \mathcal{C},
$$

where $\boldsymbol{\psi}=\left[\psi_{m}^{n, c}\right] \in \mathbb{Z}^{M \times N \times C}$ denotes the sub-channel assignment and cell association variables for different users. Moreover, $\mathbf{p} \in \mathbb{R}^{V \times N \times C}$ is the collections of power allocation variables and $P_{\max }^{c}$ is the maximum total power at the base station in cell $c$. The constraint (13b) enforces the maximum power budget in each cell. The constraint (13c) indicates that at each cell sub-channel $n$ can be allocated to at most one user. The constraint (13d) ensures that each user is only associated with one cell. Furthermore, the constraint (13e) guarantees the fixed sub-channel share of each MVNO in each cell.

\section{B. Rate-based Isolation Strategy}

The joint constrained optimization for the rate-based isolation strategy is expressed as

$$
\begin{aligned}
& \max _{\{\mathbf{p}, \boldsymbol{\psi}\}}\left\{U_{1}^{\mathrm{T}}, U_{2}^{\mathrm{T}}, \ldots, U_{v}^{\mathrm{T}}, \ldots, U_{V}^{\mathrm{T}}\right\}, \\
& \text { s.t. } \\
& \sum_{\forall v \in \mathcal{V}} \sum_{\forall n \in \mathcal{N}} \sum_{\forall m \in \mathcal{I}_{v}} \psi_{m}^{n, c} p_{v}^{n, c} \leq P_{\max }^{c}, \forall c \in \mathcal{C}, \\
& \sum_{\forall m \in \mathcal{M}} \psi_{m}^{n, c} \leq 1, \forall c \in \mathcal{C}, \forall n \in \mathcal{N}, \\
& \left(\sum_{\forall n \in \mathcal{N}} \psi_{m}^{n, c}\right)\left(\sum_{\forall n \in \mathcal{N}} \sum_{\forall c^{\prime} \neq c} \psi_{m}^{n, c^{\prime}}\right)=0, \forall c \in \mathcal{C}, \forall m \in \mathcal{M}, \\
& R_{v}^{\mathrm{T}} \geq R_{\min , v}, \forall v \in \mathcal{V}, \\
& \psi_{m}^{n, c} \in\{0,1\} \quad \forall m \in \mathcal{M}, \forall n \in \mathcal{N}, \forall c \in \mathcal{C},
\end{aligned}
$$

where the constraint (14e) represents the minimum rate requirements per MVNO. The constraint (14d) ensures that each user is only associated with one cell.
The optimization problems (13) and (14) have non-convex objective functions and involve non-linear constraints with continuous and binary variables, i.e., $\mathbf{p}$ and $\psi$. Therefore, proposing an efficient algorithm with reasonable computational complexity is desirable.

\section{PROPOSED ALGORITHM}

In this section, we will develop algorithms to find the solution for the non-convex multi-objective optimization problems (13) and (14). To achieve the Pareto optimal solution, we convert the MOOP in (13) and (14) into SOOP applying the weighted Tchebycheff method, which transforms objectives into constraints by introducing auxiliary variables $\chi_{1}$ and $\chi_{2}$. Noting that MOOP can be transformed to a SOOP through different approaches, in this paper, we use the weighted Tchebychef method which is able to find efficiently the Pareto optimal solution with a much lower complexity than other methods, e.g., the weighted product method, epsilon-method, and exponentially weighted criterion, despite the nonconvexity of the considered problem. Consequently, for resource-based isolation strategy, (13) is transformed to

$$
\begin{aligned}
& \min _{\left(\mathbf{p}, \boldsymbol{\psi}, \chi_{1}\right)} \chi_{1}, \quad \text { s.t. } \\
& \alpha_{v}\left(\Omega_{v}^{S}-U_{v}^{S}\left(\mathbf{p}_{v}, \boldsymbol{\psi}\right)\right)-\chi_{1} \leq 0, \forall v \in \mathcal{V}, \\
& (13 \mathrm{~b})-(13 \mathbf{f}),
\end{aligned}
$$

and, for rate-based isolation strategy, (14) is reformulated as

$$
\begin{aligned}
& \min _{\left(\mathbf{p}, \boldsymbol{\psi}, \chi_{2}\right)} \chi_{2}, \quad \text { s.t. } \\
& \alpha_{v}\left(\Omega_{v}^{T}-U_{v}^{T}\left(\mathbf{p}_{v}, \boldsymbol{\psi}\right)\right)-\chi_{2} \leq 0, \forall v \in \mathcal{V}, \\
& (14 \mathrm{~b})-(14 \mathbf{f}),
\end{aligned}
$$

where $\alpha_{1}, \alpha_{2}, \ldots, \alpha_{V}$ are the weighting coefficients indicating the importance of different objectives. Tchebycheff method guarantees to generate a set of Pareto-optimal solutions when $\Omega_{1}^{S}, \Omega_{2}^{S}, \ldots, \Omega_{V}^{S}$ are the utopian objective points for the resource-based isolation strategy. Noting that $\Omega_{1}^{T}, \Omega_{2}^{T}, \ldots, \Omega_{V}^{T}$ are the utopian objective points for the rate-based isolation strategy as the maximum of each objective separately [43]. The Utopia points for each MVNO can be achieved by optimizing the corresponding objective function of each MVNO $v$ given the constraints.

To deal with the joint optimization of $\mathbf{p}$ and $\psi$, two-step iterative algorithms are proposed by sequentially performing subchannel assignment and power allocation, although the two sub-problems will still remain non-convex. Despite the nonconvexity of the two sub-problems, they could be transformed as complementary geometric programming (CGP) ${ }^{2}$ and hence

\footnotetext{
${ }^{2}$ The general form of a CGP problem is as follows

$$
\begin{aligned}
& \min _{\mathbf{x}} F_{0}(\mathbf{x}) \\
& \text { s.t. } F_{i}(\mathbf{x}) \leq 1, i=1, \ldots, I
\end{aligned}
$$$$
G_{j}(x)=1, j=1, \ldots, J
$$

where $F_{0}(\mathbf{x})=f_{0}^{+}(\mathbf{x})-f_{0}^{-}(\mathbf{x}), F_{i}(\mathbf{x})=\frac{f_{i}^{+}(\mathbf{x})}{f_{i}^{-}(\mathbf{x})}, i=1, \ldots, I$ and $G_{j}(\mathbf{x})=\frac{g_{j}(\mathbf{x})}{f_{j}(\mathbf{x})}$ (in which $f_{0}^{+}$, and $f_{0}^{-}, j=0,1, \ldots, J$ are posynomial functions [44]), while $g_{j}(\mathbf{x})$ and $f_{j}(\mathbf{x})$ are posynomial and monomial functions, respectively.
} 
be solved efficiently.

\section{A. Sub-channel Assignment and Cell Association Control}

At the iteration $\tau$, with given power $\mathbf{p}_{v}(\tau)$, we attempt to find the optimal sub-channel assignment and cell association $\boldsymbol{\psi}(\tau)$ at iteration $\tau$. Accordingly, for the resource-based strategy based on (15), the association control problems can be presented as

$$
\min _{\left\{\boldsymbol{\psi}, \chi_{1}\right\}} \chi_{1}
$$

s.t.

$$
\begin{aligned}
& \sum_{\forall} \sum_{\forall v \in \mathcal{V}} \sum_{\forall n \in \mathcal{N}_{v}} \sum_{\forall m \in \mathcal{I}_{v}} \psi_{m}^{n, c} p_{v}^{n, c}(\tau) \leq P_{\max }^{c}, \forall c \in \mathcal{C} \quad \text { (17c) } \\
& \sum_{\forall m \in \mathcal{M}} \psi_{m}^{n, c} \leq 1, \forall c \in \mathcal{C}, \forall n \in \mathcal{N} \\
& \left(\sum_{\forall n \in \mathcal{N}} \psi_{m}^{n, c}\right)\left(\sum_{\forall n \in \mathcal{N}} \sum_{\forall c^{\prime} \neq c} \psi_{m}^{n, c^{\prime}}\right)=0, \forall c \in \mathcal{C}, \forall m \in \mathcal{M}
\end{aligned}
$$

$\psi_{m}^{n, c}=0, \forall m \in \mathcal{I}_{v}$ and $n \in \mathcal{N} \backslash \mathcal{N}_{v}, \forall v \in \mathcal{V}, \forall c \in \mathcal{C}$

$$
\psi_{m}^{n, c}=[0,1] \quad \forall m \in \mathcal{M}, \forall n \in \mathcal{N}, \forall c \in \mathcal{C}
$$

Since (17) is an integer non-convex and NP-hard optimization problem, hence we apply the successive convex approximation (SCA) and complementary geometric programming (CGP) to solve it with low computational complexity. First, we relax the binary constraint of sub-channel assignment and cell association indicator into continuous ones in the interval $[0,1]$, which allows us to derive a computational efficient solution. Moreover, although we have applied binary constraint relaxation to the sub-channel allocation variables, the optimal sub-channel allocation for the constraint-relaxed problem is still in binary nature, i.e., the constraint relaxation is tight. Then, we will rewrite the constraints as the form of CGP. Here, we will analyze each constraint separately. First, (17b) for any $v \in \mathcal{V}$ can be written as

$$
\frac{\alpha_{v} \Omega_{v}^{S}+\alpha_{v} \kappa_{p} \Pi_{v}^{s}\left(\mathbf{p}_{v}(\tau), \psi\right)+\alpha_{v} \kappa_{B} B_{v}^{s}(\psi)}{\chi_{1}+\alpha_{v} \kappa_{R} \sum_{\forall m \in \mathcal{I}_{v}} \sum_{\forall n \in \mathcal{N}_{v}} \sum_{\forall c \in \mathcal{C}} \psi_{m}^{n, c} \ln \left(1+\operatorname{SINRS}_{m, v}^{n, c}\right)} \leq 1
$$

To solve the CGP problem, consider $\tau_{1}$ as the iteration number. At each iteration $\tau_{1}$, the posynomial functions in the denominators of different constraints are approximated with monomial functions applying arithmetic geometric mean inequality $\left(\sum_{i=1}^{n} n_{i} a_{i} \geq \prod_{i=1}^{n} a_{i}^{n_{i}}\right)$. At the iteration $\tau_{1}$, the constraint (18) can be approximated as

$$
\begin{aligned}
& \left(\frac{\chi_{1}\left(\tau_{1}\right)}{W\left(\tau_{1}\right)}\right)^{-W\left(\tau_{1}\right)} \times \prod_{\forall m \in \mathcal{I}_{v}} \prod_{\forall n \in \mathcal{N}_{v}} \prod_{\forall c \in \mathcal{C}} \\
& \left(\frac{\alpha_{v} \kappa_{R} \psi_{m}^{n, c}\left(\tau_{1}\right) \ln \left(1+\operatorname{SINR} \mathcal{S}_{m, v}^{n, c}(\tau)\right)}{Q_{m}^{n, c}\left(\tau_{1}\right)}\right)^{-Q_{m}^{n, c}\left(\tau_{1}\right)} \times \\
& \left(\alpha_{v} \Omega_{v}^{S}+\alpha_{v} \kappa_{p} \Pi_{v}^{s}\left(\mathbf{p}_{v}(\tau), \psi\left(\tau_{1}\right)\right)+\alpha_{v} \kappa_{B} B_{v}^{s}\left(\psi\left(\tau_{1}\right)\right)\right) \leq 1
\end{aligned}
$$

where

$$
W\left(\tau_{1}\right)=\frac{\chi_{1}\left(\tau_{1}-1\right)}{\chi_{1}\left(\tau_{1}-1\right)+\alpha_{v} \kappa_{R} R_{v}^{s}\left(\mathbf{p}_{v}(\tau), \psi\left(\tau_{1}-1\right)\right)}
$$

and

$$
Q_{m}^{n, c}\left(\tau_{1}\right)=\frac{\alpha_{v} \kappa_{R} \psi_{m}^{n, c}\left(\tau_{1}-1\right) \ln \left(1+\operatorname{SINR}_{m, v}^{n, c}(t)\right)}{\chi_{1}\left(\tau_{1}-1\right)+\alpha_{v} \kappa_{R} R_{v}^{s}\left(\mathbf{p}_{v}(\tau), \psi\left(\tau_{1}-1\right)\right)} .
$$

Furthermore, to have a standard GP formulation, the constraint (17e) needs to be relaxed to guarantee the equality constraint only involves monomial functions. In the following, we first relax this constraint and then apply iterative arithmetic geometric mean approximation (AGMA) algorithm to get the monomial approximation. At iteration $\tau_{1}$, we define $x_{m, c}\left(\tau_{1}\right)=\sum_{n \in \mathcal{N}} \psi_{m}^{n, c}\left(\tau_{1}\right)$ and $y_{m}\left(\tau_{1}\right)=$ $\sum_{c \in \mathcal{C}} \sum_{n \in \mathcal{N}} \psi_{m}^{n, c}\left(\tau_{1}\right)$, hence the constraint (17e) can be rewritten as

$$
x_{m, c}\left(\tau_{1}\right)\left(y_{m}\left(\tau_{1}\right)-x_{m, c}\left(\tau_{1}\right)\right)=0
$$

which is not a monomial function. (22) can be transformed into $x_{m, c}\left(\tau_{1}\right) y_{m}\left(\tau_{1}\right)=x_{m, c}^{2}\left(\tau_{1}\right)$ and by adding 1 to both the left and right hand sides, we have $x_{m, c}\left(\tau_{1}\right) y_{m}\left(\tau_{1}\right)+1=$ $x_{m, c}^{2}\left(\tau_{1}\right)+1$ for all $c \in \mathcal{C}, m \in \mathcal{M}$. We introduce an auxiliary variable $s_{m, c}\left(\tau_{1}\right) \geq 0$ to relax and convert (22) into the posynomial inequalities as follows

$$
x_{m, c}\left(\tau_{1}\right) y_{m}\left(\tau_{1}\right)+1 \leq s_{m, c}\left(\tau_{1}\right) \leq x_{m, c}^{2}\left(\tau_{1}\right)+1 .
$$

The above inequalities can be written as

$$
\begin{aligned}
& x_{m, c}\left(\tau_{1}\right) y_{m}\left(\tau_{1}\right) s_{m, c}^{-1}\left(\tau_{1}\right)+s_{m, c}^{-1}\left(\tau_{1}\right) \leq 1, \\
& \frac{s_{m, c}\left(\tau_{1}\right)}{x_{m, c}^{2}\left(\tau_{1}\right)+1} \leq 1
\end{aligned}
$$

Hence, (24) is a posynomial inequality constraint already, although (25) needs to be transformed using AGMA approximation as

$$
\left[\frac{1}{\lambda_{m, c}\left(\tau_{1}\right)}\right]^{-\lambda_{m, c}\left(\tau_{1}\right)} s_{m, c}\left(\tau_{1}\right) \times\left[\frac{x_{m, c}^{2}\left(\tau_{1}\right)}{\alpha_{m, c}\left(\tau_{1}\right)}\right]^{-\alpha_{m, c}\left(\tau_{1}\right)} \leq 1
$$

where $\lambda_{m, c}\left(\tau_{1}\right)=\frac{1}{x_{m, c}^{2}\left(\tau_{1}-1\right)+1}$ and $\quad \alpha_{m, c}\left(\tau_{1}\right)=$ $\frac{x_{m, c}^{2}\left(\tau_{1}-1\right)}{x_{m, c}^{2}\left(\tau_{1}-1\right)+1}$. In addition, the equality constraints $x_{m, c}\left(\tau_{1}\right)=$ $\sum_{n \in \mathcal{N}} \psi_{m}^{n, c}\left(\tau_{1}\right)$ and $y_{m}\left(\tau_{1}\right)=\sum_{c \in \mathcal{C}} \sum_{n \in \mathcal{N}} \psi_{m}^{n, c}\left(\tau_{1}\right)$ also need to be converted into the monomial functions by applying AGMA approximation as

$$
\begin{aligned}
& x_{m, c}\left(\tau_{1}\right) \prod_{n \in \mathcal{N}}\left[\frac{\psi_{m}^{n, c}\left(\tau_{1}\right)}{\zeta_{m}^{n, c}\left(\tau_{1}\right)}\right]^{-\zeta_{m}^{n, c}\left(\tau_{1}\right)}=1, \\
& y_{m}\left(\tau_{1}\right) \prod_{c \in \mathcal{C}, n \in \mathcal{N}}\left[\frac{\psi_{m}^{n, c}\left(\tau_{1}\right)}{\eta_{m}^{n, c}\left(\tau_{1}\right)}\right]^{-\eta_{m}^{n, c}\left(\tau_{1}\right)}=1,
\end{aligned}
$$

where $\zeta_{m}^{n, c}\left(\tau_{1}\right)=\frac{\psi_{m}^{n, c}\left(\tau_{1}-1\right)}{\sum_{n \in \mathcal{N}} \psi_{m}^{n, c}\left(\tau_{1}-1\right)} \quad$ and $\quad \eta_{m}^{n, c}\left(\tau_{1}\right)=$ $\frac{\psi_{m}^{n, c}\left(\tau_{1}-1\right)}{\sum_{c \in \mathcal{C}} \sum_{n \in \mathcal{N}} \psi_{m}^{n, c}\left(\tau_{1}-1\right)}$.

Using the approximated constraints, at each iteration $\tau_{1}$, (18) can be locally approximated by a GP problem. Iteratively 
solving the GP approximations, using SCA method, the algorithm will reach to the sub-channel and association control solution.

Similarly, in the cased of rate-based strategy, we will use sequential GP approximation to solve the non-convex optimization problem (16), i.e.,

$\min _{\left\{\boldsymbol{\psi}, \chi_{2}\right\}} \chi_{2}$

s.t.

$$
\begin{aligned}
& \sum_{v}\left(\Omega_{v}^{T}-U_{v}^{T}\left(\mathbf{p}_{v}(\tau), \boldsymbol{\psi}\right)\right)-\chi_{2} \leq 0, \forall v \in \mathcal{V}, \\
& \sum_{\forall v \in \mathcal{V} \forall n \in \mathcal{N}_{v}} \psi_{m}^{n, c} p_{v}^{n, c}(\tau) \leq P_{\max }^{c}, \forall c \in \mathcal{C}, \\
& \left(\sum_{m \in \mathcal{M}}^{n, c} \leq 1, \forall c \in \mathcal{C}, \forall n \in \mathcal{N},\right. \\
& \left(\sum_{\forall n \in \mathcal{N}} \psi_{m}^{n, c}\right)\left(\sum_{\forall n \in \mathcal{N}} \sum_{\forall c^{\prime} \neq c} \psi_{m}^{n, c^{\prime}}\right)=0, \forall c \in \mathcal{C}, \forall m \in \mathcal{M},
\end{aligned}
$$

$$
\begin{aligned}
& R_{v}^{T} \geq R_{\min , v}, \forall v \in \mathcal{V}, \\
& \psi_{m}^{n, c}=[0,1], \quad \forall m \in \mathcal{M}, \forall n \in \mathcal{N}, \forall c \in \mathcal{C} .
\end{aligned}
$$

Apparently, for constraints (29b) and (29e), the same approach as for (17b) and (17e) can be applied to convert them into the CGP form. There is only minimum rate requirement constraint (29f) that can be transformed into

$R_{\min , v} \prod_{\forall m \in \mathcal{I}_{v}} \prod_{\forall n \in \mathcal{N}} \prod_{\forall c \in \mathcal{C}}\left(\frac{\psi_{m}^{n, c}\left(\tau_{1}\right) R_{v}^{T}\left(\mathbf{p}_{v}(\tau)\right)}{\varphi_{m}^{n, c}\left(\tau_{1}\right)}\right)^{-\varphi_{m}^{n, c}\left(\tau_{1}\right)} \leq 1$

where $\varphi_{m}^{n, c}\left(\tau_{1}\right)=\frac{\psi_{m}^{n, c}\left(\tau_{1}-1\right) R_{v}^{T}\left(\mathbf{p}_{v}(\tau)\right)}{\sum_{\forall m \in \mathcal{I}_{v}} \sum_{\forall n \in \mathcal{N}} \sum_{\forall c \in \mathcal{C}} \psi_{m}^{n, c}\left(\tau_{1}-1\right) R_{v}^{T}\left(\mathbf{p}_{v}(\tau)\right)}$.

Having the posynomial constraint (29), (30) can be locally approximated and replaced by it GP counterpart. Subsequently, adapting a SCA technique, the solution of the sub-channel assignment and association control can be achieved.

\section{B. Power Allocation}

After sub-channel assignment and cell association at iteration $\tau$, we need to solve the non-convex power allocation problem based on (15) and (16). Here, we use an approach based on the Difference of Convex (DC) programming problem [45].

Motivated by the special structure of the formulated of problem of interest, in particular (15b) and (16b) in Difference of Convex functions form as follows:

$$
f(\mathbf{p})-g(\mathbf{p})
$$

where $f(\mathbf{p})$ and $g(\mathbf{p})$ are defined as

$$
\begin{aligned}
f(\mathbf{p}) & =\kappa_{\mathrm{R}} \sum_{\forall m \in \mathcal{I}_{v}} \sum_{\forall n \in \mathcal{N}} \sum_{\forall c \in \mathcal{C}} \psi_{m}^{n, c} \ln \left(N_{0}+\sum_{\forall c^{\prime}} p_{v}^{n, c^{\prime}} h_{m}^{n, c^{\prime}}\right), \quad \text { gene } \\
g(\mathbf{p}) & =\kappa_{\mathrm{R}} \sum_{\forall m \in \mathcal{I}_{v}} \sum_{\forall n \in \mathcal{N}} \sum_{\forall c \in \mathcal{C}} \psi_{m}^{n, c} \ln \left(N_{0}+\sum_{\forall c^{\prime} \neq c} p_{v}^{n, c^{\prime}} h_{m}^{n, c^{\prime}}\right) \\
& +\kappa_{P} \sum_{\forall m \in \mathcal{I}_{v}} \sum_{\forall n \in \mathcal{N}} \sum_{\forall c \in \mathcal{C}} \psi_{m}^{n, c} p_{v}^{n, c}+\kappa_{B} \sum_{\forall m \in \mathcal{I}_{v}} \sum_{\forall n \in \mathcal{N}} \sum_{\forall c \in \mathcal{C}} \psi_{m}^{n, c},
\end{aligned}
$$

$\overline{\text { Algorithm } 1 \text { Iterative Joint cell association and power alloca- }}$ tion algorithm.

1: Initialization: Set $\tau=0$, and $P_{v}^{n, c}(\tau)$ and $\psi_{m}^{n, c}(\tau)$

2: Repeat Set $\tau=\tau+1$

3: Step 1. A cell association and channel assignment

4: Initalization for Step 1.A:

5: Set $\tau_{1}=0, \psi_{m}^{n, c}\left(\tau_{1}\right)=\psi_{m}^{n, c}(\tau), P_{v}^{n, c}\left(\tau_{1}\right)=P_{v}^{n, c}(\tau)$ and set arbitrary inital for $s_{m, c}\left(\tau_{1}\right)$.

6: Repeat: Set $\tau_{1}=\tau_{1}+1$

7: $\quad$ Step 1.A.1 Update $W\left(\tau_{1}\right), Q\left(\tau_{1}\right), \lambda_{m, c}\left(\tau_{1}\right), \alpha_{m, c}\left(\tau_{1}\right)$, $\zeta_{m}^{n, c}\left(\tau_{1}\right)$ and $\eta_{m}^{n, c}\left(\tau_{1}\right)$.

8: Step 1.A.2 Find the optimal cell association $\psi_{m}^{n, c}$ in (10) using CVX.

9: Until $\left\|\psi^{*}\left(\tau_{1}\right)-\psi^{*}\left(\tau_{1}-1\right)\right\| \leq \epsilon_{1}$

10: Step 1.B Power allocation

11: Initalization for Step 1.B: Set $\tau_{2}=0, P_{v}^{n, c}\left(\tau_{2}\right)=$ $P_{v}^{n, c}(\tau)$

12: $\quad$ Repeat: Set $\tau_{2}=\tau_{2}+1$

13: Step 1.B.1 Initialize $\mathbf{x}^{0}$, set iteration number $k=0$.

14: while

15: Define convex approximation of $q^{(k)}(\mathbf{z})$ as $\hat{q}^{k}(\mathbf{x})=f(\mathbf{x})-g\left(\mathbf{x}^{(k)}\right)-\nabla g^{T}\left(\mathbf{x}^{(k)}\right)\left(\mathbf{x}-\mathbf{x}^{(k)}\right)$

16:

17: Solve the convex problem

$$
\text { 18: } \quad \mathbf{x}^{(k+1)}=\arg \min _{\mathbf{x} \in \mathcal{X}} \hat{q}^{(k)}(\mathbf{x})
$$

19: $\quad k \leftarrow k+1$

\section{0: end while}

21: $\quad$ Step 1.B.2 Find the optimal power allocation according to $\mathrm{DC}$ algorithm

22: Until $\left\|P^{*}\left(\tau_{2}\right)-P^{*}\left(\tau_{2}-1\right)\right\| \leq \epsilon_{2}$

23: $\quad P(\tau)=P\left(\tau_{2}\right)$.

24: Until $\left\|\psi^{*}(\tau)-\psi^{*}(\tau-1)\right\| \leq \epsilon_{1}$ and $\| P^{*}(\tau)-P^{*}(\tau-$ 1) $\| \leq \epsilon_{2}$

$f(\mathbf{p})-g(\mathbf{p})$ are D.C. functions. To obtain the Taylor series of $g(\mathbf{p})$ we need their derivatives which can be derived as follows:

$$
\nabla g(\mathbf{p})_{n, k, i}=\frac{\partial g(\mathbf{p})}{\partial g_{k, i}^{n}}
$$

In this technique, $g(\mathbf{p})$ is estimated with the first order approximation as $g\left(\mathbf{p}^{(t)}\right)+\left\langle\nabla g\left(\mathbf{p}^{(t)}\right),\left(\mathbf{p}-\mathbf{p}^{(t)}\right)\right\rangle$ at point $\mathbf{p}^{(t)}$ to convexify the function. Here, $\langle\mathbf{X}, \mathbf{Y}\rangle$ denotes the standard inner product on $\mathbf{R}^{N \times M}$. The feasible points $\mathbf{p}^{(0)}$ as the first iteration are selected and then the next iterations will be generated to obtain the solution of the following problem:

$$
\begin{aligned}
& \min _{\{\mathbf{p}, \chi\}} \chi_{1}, \\
& \text { s.t. } \\
& \alpha_{v}\left(\Omega_{v}-\left(f(\mathbf{p})-g\left(\mathbf{p}^{(t)}\right)\right.\right. \\
&\left.\left.\quad-\left\langle\nabla g\left(\mathbf{p}^{(t)}\right),\left(\mathbf{p}-\mathbf{p}^{(t)}\right)\right\rangle\right)\right)-\chi_{1} \leq 0,
\end{aligned}
$$




\section{Joint Cell Association and Power Allocation Algorithm}

Our iterative algorithm is summarized in Algorithm 1. This algorithm starts with obtaining a feasible solution for subchannel and transmit power. In each iteration, this algorithm alternatively find sub-channel assignment and cell association of users in MVNOs and allocates power to them at base stations. The iterations continue until no further improvement can be made.

\section{Computational Complexity and Convergence Analysis of Algorithm 1}

1) Computational Complexity: In Algorithm 1, to solve the original optimization problems in (17) and (29), the problem is transformed into a GP approximation and a series of GP problems is solved iteratively until convergence. A GP problem is solved using an interior point method in CVX. According to [46], the required number of iterations (Newton steps) to solve by this method is $\frac{\log \left(c /\left(t^{0} \delta\right)\right)}{\log (\xi)}$, where $c$ is the total number of constraints, $t^{0}$ is the initial point used by the solver in CVX to apply the interior point method, $0<\delta \leq 1$ is the stopping criterion, and $\xi$ is used for updating the accuracy of the method.

Regarding the resource-based isolation method, the total number of constraints of (17) is $c_{1, s}=C+N C+2 M N C+$ $V+3 M C+M$ for Step 1.A and $c_{2, s}=V+C$ for Step 1.B. For each iteration, a Newton step of an interior-point method costs $\mathcal{O}\left(c \varrho^{2}\right)$ operations where $c$ is the number of constraints and $\varrho$ is the number of variables. For the optimization problem of (17) for Step 1.A, the total number of variables is $\varrho_{1, s}=4 M N C+4 M C+M+2=\mathcal{O}(M N C)$. Thus, the total computational complexity of solving the GP problem of Step 1.A (i.e., number of Newton steps $\times$ required operations per step) is polynomial, namely, $C_{1}^{\mathrm{GP}}=\mathcal{O}\left(c_{1, s}{ }^{1.5} \varrho_{1, s}^{2}\right)=$ $\mathcal{O}\left(M^{3.5} V^{1.5} N^{3.5} C^{3.5}\right)$. In addition, the total number of variable for Step 1.B is $\varrho_{2, s}=M N C+N V C+1$. Hence, the computational complexity of solving the DC algorithm of Step 1.B is $C_{1}^{D C}=\mathcal{O}\left(c_{2, s}{ }^{1.5} \varrho_{2, s}^{2}\right)=\mathcal{O}\left(M^{2} V^{3.5} N^{2} C^{3.5}\right)$.

On the other hand, for the rate-based isolation method by utilizing the same approach, the total number of constraints of (29) is $c_{1, t}=C+N C+M N C+2 V+3 M C+M$ for Step 1.A, and $c_{2, t}=2 V+C$ for Step 1.B. Also the number of variables is $\varrho_{1, t}=5 M N C+4 M C+M+2$ and $\varrho_{2, t}=M N C+N V C+1$, hence, the computational complexity becomes $C_{2}^{\mathrm{GP}}=\mathcal{O}\left(c_{1, s}{ }^{1.5} \varrho_{1, s}^{2}\right)=\mathcal{O}\left(M^{3.5} V^{1.5} N^{3.5} C^{3.5}\right)$ and $C_{1}^{D C}=\mathcal{O}\left(c_{2, s}{ }^{1.5} \varrho_{2, s}^{2}\right)=\mathcal{O}\left(M^{2} V^{3.5} N^{2} C^{3.5}\right)$, respectively. In addition to computations required to solve the GP problems in each iteration, additional computational complexity is incurred by applying AGMA approximations. For Algorithm 1 of Step 1.A, the number of computations required for conversion to the GP form using AGMA is $i=5 M N C+4 M C+M$ which is of lower order than $C_{1}^{\mathrm{GP}}$. Therefore, the total computational complexity of Algorithm 1 can be calculated as the number of iterations until convergence multiplied by $C_{1}^{\mathrm{GP}}=\mathcal{O}\left(c^{1.5} \varrho^{2}\right)=\mathcal{O}\left(M^{3.5} V^{2} N^{3.5} C^{1.5}\right)$. Based on numerical results, the number of iterations until convergence of Algorithm 1 is plotted versus the number of users, $M$, and sub-carriers, $N$ in Fig. 2, respectively. It

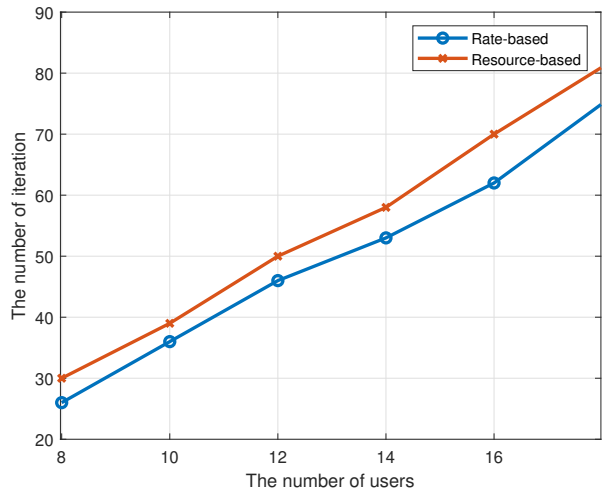

(a) Number of iterations until convergence versus number of users

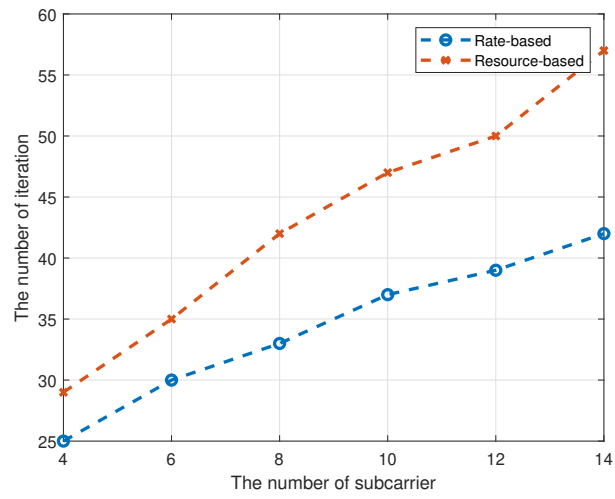

(b) Number of iterations until convergence versus number of subcarriers

Fig. 2. Convergence Behaviours

has been shown that as $M$ and $N$ increase the required number of iterations for each problem to converge increases but only linearly. Consequently, we can deduce that the overall complexity of GP for Algorithm 1 only grows polynomially with the number of users and subcarriers.

2) Convergence Analysis:

Proposition 1: With AGMA, Step 1.A converges to a local optimal solution that satisfies the KKT conditions of the original problem.

Proof: In [47], it is shown that the conditions for the convergence of the SCA are satisfied and guarantee that solutions of series of approximation by AGMA converges to a point that satisifies the KKT conditions of optimization problem, i.e., a local maximum is attained [48].

Proposition 2: With DC approximation, the SCA approach generates a sequence of improved feasible solutions, which will finally converge to a locally optimal solution $\mathbf{p}^{*}$ of Step 1.B.

Proof: We notice that at the conclusion of iteration $\tau_{2}>0$, it must be true that

$$
\begin{aligned}
\alpha_{v}\left(\Omega_{v}-\left(f\left(\mathbf{p}^{\tau_{2}}\right)-g\left(\mathbf{p}^{\left(\tau_{2}-1\right)}\right)\right.\right. & \\
& \left.\left.-\left\langle\nabla g\left(\mathbf{p}^{\left(\tau_{2}-1\right)}\right),\left(\mathbf{p}^{\tau_{2}}-\mathbf{p}^{\left(\tau_{2}-1\right)}\right)\right\rangle\right)\right)-\chi_{1} \leq 0,
\end{aligned}
$$

On the other hand, the gradient of $g_{m}\left(\mathbf{p}^{\left(\tau_{2}\right)}\right)$ is indeed its 
supergradient [49] since $g_{m}\left(\mathbf{p}^{\left(\tau_{2}\right)}\right)$ is concave, i.e., $g_{m}\left(\mathbf{p}^{\tau_{2}}\right) \leq$ $g_{m}\left(\mathbf{p}^{\left(\tau_{2}-1\right)}\right)+\nabla\left\langle g_{m}\left(\mathbf{p}^{\left(\tau_{2}-1\right)}\right),\left(\mathbf{p}-\mathbf{p}^{\left(\tau_{2}\right)}\right)\right\rangle$. It follows that

$$
g_{0}\left(\mathbf{p}^{\left(\tau_{2}-1\right)}\right)+\left\langle\nabla g_{0}\left(\mathbf{p}^{\left(\tau_{2}-1\right)}\right),\left(\mathbf{p}-\mathbf{p}^{\left(\tau_{2}\right)}\right)\right\rangle \geq g_{0}\left(\mathbf{p}^{\tau_{2}}\right)
$$

From (36) and (37), it can be deduced that $\alpha_{v}\left(\Omega_{v}-\left(f\left(\mathbf{p}^{\tau_{2}}\right)-g\left(\mathbf{p}^{\left(\tau_{2}\right)}\right)\right)\right)-\chi_{1} \leq 0$. That is, the optimal solution generated at each iteration is always feasible with respect to problem. The objective value of (35) is either unchanged or improved after iteration $\tau_{2}$. Since the constraint set is compact and each subproblem remains within the feasible region of (35), the SCA approach will converge to a locally optimal solution $\mathbf{p}^{*}$ of (35), i.e., a local maximum is obtained.

Proposition 3: For a feasible problem of two objective functions, Algorithm 1 will converge to a local maximum.

Proof: Denote the objective functions of (17) as

$$
\Psi(\mathbf{p}, \psi):=\chi_{1}
$$

We have relationship

$$
\begin{aligned}
\Psi(\mathbf{p}(\tau), \boldsymbol{\psi}(\tau)) & =\min _{\left(\boldsymbol{\psi}, \chi_{1}\right)} \Psi(\mathbf{p}(\tau), \boldsymbol{\psi}) \\
& \leq \Psi(\mathbf{p}(\tau), \boldsymbol{\psi}(\tau-1)) \\
& =\min _{\left(\mathbf{p}, \chi_{1}\right)} \Psi(\mathbf{p}(\tau), \boldsymbol{\psi}(\tau-1)) \\
& \leq \Psi(\mathbf{p}(\tau-1), \boldsymbol{\psi}(\tau-1))
\end{aligned}
$$

Essentially, this means that Alg. 1 gives nonincreasing functions as the iterations continue. With power constraints, Alg. 1 is guaranteed to converge to a locally optimal solution $\left(\boldsymbol{\psi}^{o p t}, \mathbf{p}^{o p t}\right)$, which corresponds to a local maximum of (35).

\section{MONOTONIC OPTIMIZATION METHOD}

Many non-convex problems to optimize communication and networking systems have monotonicity or hidden monotonicity structures. Efficient use of the monotonicity properties would considerably reduce the difficulty of obtaining the global optimal solutions of problems, and this is indeed a vital thought underlying the theory of monotonic optimization.

The canonical monotonic optimization is concerned with problems of the following form

$$
\max \{f(\mathbf{x}) \mid \mathbf{x} \in \mathcal{G} \cap \mathcal{H}\}
$$

where $f(x): \mathcal{R}_{+}^{n} \rightarrow \mathcal{R}$ is an increasing function, $G \subset$ $[\mathbf{0}, \mathbf{b}] \subset \mathcal{R}_{+}^{n}$ is compact normal set with noempty interior, and $\mathcal{H}$ is a closed conormal set on $[\mathbf{0}, \mathbf{b}]$. Sometimes, $\mathcal{H}$ is not present in the formulation, and the problem becomes

$$
\max \{f(\mathbf{x}) \mid \mathbf{x} \in \mathcal{G}\}
$$

In this case, we can assume that the conormal set $\mathcal{H}$ in (41) is box $[\mathbf{0}, \mathbf{b}]$ itself. In the remaining of this monograph, we assume that the considered problem is feasible, i.e., $\mathcal{G} \cap \mathcal{H} \neq \emptyset$

In real applications, sets $\mathcal{G}$ and $\mathcal{H}$ often result from constraints involving increasing functions $g_{i}(\mathbf{x}): \mathcal{R}_{+}^{n} \rightarrow \mathcal{R}$ and $h_{i}(\mathbf{x}): \mathcal{R}_{+}^{n} \rightarrow \mathcal{R}$

$$
\begin{array}{r}
g_{i}(\mathbf{x}) \leq 0, \quad i=1, \ldots, m_{1}, \\
h_{i}(\mathbf{x}) \geq 0, \quad i=m_{1}+1, \ldots, m .
\end{array}
$$

Setting $g(\mathbf{x})=\max \left\{g_{1}(\mathbf{x}), \ldots, g_{m_{1}}(\mathbf{x})\right\}$ and $h(\mathbf{x})=$ $\min \left\{h_{m_{1}+1}(\mathbf{x}), \ldots, h_{m}(\mathbf{x})\right\}$, the above inequalities are equivalent to

$$
g(\mathbf{x}) \leq 0, \quad h(\mathbf{x}) \geq 0
$$

In practice, $f(\mathbf{x})$ would be a certain system performance, $g(\mathbf{x})$ would be certain resources with limited availability, and $h(\mathbf{x})$ would be the level of users' satisfaction. Overall, the limitations of real systems can lead to a feasible set of arbitrary shapes instead of the one properly formulated in (41).

\section{A. Difference of Increasing Functions in the Constraints}

We consider the problem

$$
\max \left\{f(\mathbf{x}) \mid g(\mathbf{x})-h(\mathbf{x}) \leq 0, \mathbf{x} \in \Omega \subset \mathcal{R}_{+}^{n}\right\}
$$

where $f, g, h$ are increasing and continuous functions on $\mathcal{R}_{+}^{n}$, and $\Omega$ is a normal set contained in $[\mathbf{0}, \mathbf{b}] \subset \mathcal{R}_{+}^{n}$. To convert the problem into the canonical, we can divide the inequality $g(\mathbf{x})-h(\mathbf{x}) \leq 0$ for $\mathbf{x} \in \Omega$ into two inequalities:

$$
g(\mathbf{x})+t \leq g(\mathbf{b}), h(\mathbf{x})+t \geq g(\mathbf{b})
$$

where $t \geq 0$. Define

$$
\begin{gathered}
\mathcal{G}=\left\{(\mathbf{x}, t) \in \mathcal{R}_{+}^{n} \times \mathcal{R}_{+} \mid \mathbf{x} \in \Omega, g(\mathbf{x})+t \leq g(\mathbf{b}),\right. \\
\quad 0 \leq t \leq g(\mathbf{b})-g(\mathbf{0})\} \\
\mathcal{H}=\left\{(\mathbf{x}, t) \in \mathcal{R}_{+}^{n} \times \mathcal{R}_{+} \mid h(\mathbf{x})+t \geq g(\mathbf{b})\right\}
\end{gathered}
$$

Therefore, the problem is monotonic optimization in canonical form.

According to this properties of monotonic algorithm, we will transform the ordinary problems (non-monotonic) constraints into the monotonic form.

\section{B. SOOP Convert into Monotonic Optimiation}

1) Resource-based isolation Strategy: To reduce the complexity, first the SOOP of (17) need to convert into the maximization optimization problem. Assume $Z_{1}=-\chi_{1}$, hence the objective problem will be

$$
\begin{aligned}
& \max _{\left(\mathbf{p}, \boldsymbol{\psi}, Z_{1}\right)} Z_{1} \\
& \text { s.t. } \\
& Z_{1} \leq \alpha_{v}\left(U_{v}^{S}\left(\mathbf{p}_{v}, \boldsymbol{\psi}\right)-\Omega_{v}^{S}\right), \forall v \in \mathcal{V}, \\
& \sum_{\forall v \in \mathcal{V}} \sum_{\forall n \in \mathcal{N}_{v}} \sum_{\forall m \in \mathcal{I}_{v}} \psi_{m}^{n, c} p_{v}^{n, c} \leq P_{\max }^{c}, \forall c \in \mathcal{C} \\
& \sum_{\forall m \in \mathcal{M}} \psi_{m}^{n, c} \leq 1, \forall c \in \mathcal{C}, \forall n \in \mathcal{N}, \\
& \left(\sum_{\forall n \in \mathcal{N}} \psi_{m}^{n, c}\right)\left(\sum_{\forall n \in \mathcal{N}} \sum_{\forall c^{\prime} \neq c} \psi_{m}^{n, c^{\prime}}\right)=0, \forall c \in \mathcal{C}, \forall m \in \mathcal{M} \\
& \psi_{m}^{n, c}=0, \forall m \in \mathcal{I}_{v} \text { and } n \in \mathcal{N} \backslash \mathcal{N}_{v}, \forall v \in \mathcal{V} \\
& \psi_{m}^{n, c} \in\{0,1\} \quad \forall m \in \mathcal{M}, \forall n \in \mathcal{N}
\end{aligned}
$$

Note that this optimization problem is a mixed-integer nonlinear programming. And it is not a monotonic optimization problem in canonical form since (47b) and $(47 \mathrm{~g})$ are not monotonic. 
Then we will transfer the non-monotonic constraints into monotonic constraints form. To facilitate the presentation, the constraints (47b) can be rewritten as

$$
\begin{aligned}
& U_{v}^{S}\left(\mathbf{p}_{v}, \boldsymbol{\psi}\right) \geq \Omega_{v}^{S}+\frac{Z_{1}}{\alpha_{v}} \\
& \kappa_{\mathrm{R}} R_{v}^{\mathrm{S}}\left(\mathbf{p}_{v}, \boldsymbol{\psi}\right)-\kappa_{\mathrm{P}} \Pi_{v}^{\mathrm{S}}\left(\mathbf{p}_{v}, \boldsymbol{\psi}\right)-\kappa_{\mathrm{B}} B_{v}^{\mathrm{S}}(\boldsymbol{\psi}) \geq \Omega_{v}^{S}+\frac{Z_{1}}{\alpha_{v}}
\end{aligned}
$$

where the achievable rate of all users is expressed as

$$
\begin{aligned}
& R_{v}^{\mathrm{S}}\left(\mathbf{p}_{v}, \psi\right)=\sum_{\forall m \in \mathcal{I}_{v}} \sum_{\forall n \in \mathcal{N}_{v}} \sum_{\forall c \in \mathcal{C}} \psi_{m}^{n, c} \ln \left(1+\operatorname{SINR} \mathcal{S}_{m, v}^{n, c}\right) \\
& =\sum_{\forall m \in \mathcal{I}_{v}} \sum_{\forall n \in \mathcal{N}_{v}} \sum_{\forall c \in \mathcal{C}} \psi_{m}^{n, c} \ln \left(1+\frac{p_{v}^{n, c} h_{m}^{n, c}}{N_{0}+\sum_{\forall c^{\prime} \neq c} p_{v}^{n, c^{\prime}} h_{m}^{n, c^{\prime}}}\right) \\
& =\sum_{\forall m \in \mathcal{I}_{v}} \sum_{\forall n \in \mathcal{N}_{v}} \sum_{\forall c \in \mathcal{C}} \ln \left(N_{0}+\sum_{\forall c} \psi_{m}^{n, c} p_{v}^{n, c} h_{m}^{n, c}\right) \\
& -\sum_{\forall m \in \mathcal{I}_{v}} \sum_{\forall n \in \mathcal{N}_{v}} \sum_{\forall c \in \mathcal{C}} \ln \left(N_{0}+\sum_{\forall c^{\prime} \neq c} \psi_{m}^{n, c^{\prime}} p_{v}^{n, c^{\prime}} h_{m}^{n, c^{\prime}}\right)
\end{aligned}
$$

The total transmit power is

$$
\Pi_{v}^{\mathrm{S}}\left(\mathbf{p}_{v}, \boldsymbol{\psi}\right)=\sum_{\forall m \in \mathcal{I}_{v}} \sum_{\forall n \in \mathcal{N}_{v}} \sum_{\forall c \in \mathcal{C}} \psi_{m}^{n, c} p_{v}^{n, c}
$$

and the total bandwidth is

$$
B_{v}^{\mathrm{S}}(\boldsymbol{\psi})=\sum_{\forall m \in \mathcal{I}_{v}} \sum_{\forall n \in \mathcal{N}_{v}} \sum_{\forall c \in \mathcal{C}} \psi_{m}^{n, c}
$$

We assume $q_{m, v}^{n, c}=\psi_{m}^{n, c} p_{v}^{n, c}$, hence the constraints (47b) become

$$
\begin{aligned}
& \Omega_{v}+\frac{Z_{1}}{\alpha_{v}}+\kappa_{\mathrm{R}} \sum_{\forall m \in \mathcal{I}_{v}} \sum_{\forall n \in \mathcal{N}_{v}} \sum_{\forall c \in \mathcal{C}} \ln \left(N_{0}+\sum_{\forall c^{\prime} \neq c} q_{m, v}^{n, c^{\prime}} h_{m}^{n, c^{\prime}}\right) \\
& +\kappa_{\mathrm{P}} \sum_{\forall m \in \mathcal{I}_{v}} \sum_{\forall n \in \mathcal{N}_{v}} \sum_{\forall c \in \mathcal{C}} q_{m, v}^{n, c}+\kappa_{\mathrm{B}} \sum_{\forall m \in \mathcal{I}_{v}} \sum_{\forall n \in \mathcal{N}_{v}} \sum_{\forall c \in \mathcal{C}} \psi_{m}^{n, c} \\
& -\kappa_{\mathrm{R}} \sum_{\forall m \in \mathcal{I}_{v}} \sum_{\forall n \in \mathcal{N}_{v}} \sum_{\forall c \in \mathcal{C}} \ln \left(N_{0}+\sum_{\forall c} q_{m, v}^{n, c} h_{m}^{n, c}\right) \leq 0
\end{aligned}
$$

In order to have $g\left(q_{m, v}^{n, c}, \psi_{m}^{n, c}\right)-h\left(q_{m, v}^{n, c}\right) \leq 0$, where

$$
\begin{aligned}
& g\left(q_{m, v}^{n, c}, \psi_{m}^{n, c}\right)=\Omega_{v}+\frac{Z_{1}}{\alpha_{v}} \\
& +\kappa_{\mathrm{R}} \sum_{\forall m \in \mathcal{I}_{v}} \sum_{\forall n \in \mathcal{N}_{v}} \sum_{\forall c \in \mathcal{C}} \ln \left(N_{0}+\sum_{\forall c^{\prime} \neq c} q_{m, v}^{n, c^{\prime}} h_{m}^{n, c^{\prime}}\right) \\
& +\kappa_{\mathrm{P}} \sum_{\forall m \in \mathcal{I}_{v}} \sum_{\forall n \in \mathcal{N}_{v}} \sum_{\forall c \in \mathcal{C}} q_{m, v}^{n, c}+\kappa_{\mathrm{B}} \sum_{\forall m \in \mathcal{I}_{v}} \sum_{\forall n \in \mathcal{N}_{v}} \sum_{\forall c \in \mathcal{C}} \psi_{m}^{n, c} \\
& h\left(q_{m, v}^{n, c}\right)=\kappa_{\mathrm{R}} \sum_{\forall m \in \mathcal{I}_{v}} \sum_{\forall n \in \mathcal{N}_{v}} \sum_{\forall c \in \mathcal{C}} \ln \left(N_{0}+\sum_{\forall c} q_{m, v}^{n, c} h_{m}^{n, c}\right)
\end{aligned}
$$

where $h\left(q_{m, v}^{n, c}\right)$ is an increasing function. In (51), since $\psi_{m}^{n, c}$ is a binary variable $g\left(q_{m, v}^{n, c}, \psi_{m}^{n, c}\right)$ becomes an increasing function as well. These constraints becomes the difference of increasing functions in the constraints. To transform the problem into the canonical form, we can split the inequality into two inequalities by introducing a slack variable $t$ :

$$
g\left(q_{m, v}^{n, c}, \psi_{m}^{n, c}\right)+t \leq g(\mathbf{b}), \quad h\left(q_{m, v}^{n, c}\right)+t \geq g(\mathbf{b})
$$

In order to transform the constraint (13d), we define $x_{m, c}=\sum_{n \in \mathcal{N}} \psi_{m}^{n, c}$ and $y_{m}=\sum_{c \in \mathcal{C}} \sum_{n \in \mathcal{N}} \psi_{m}^{n, c}$, hence the constraint can be rewritten as

$$
x_{m, c}\left(y_{m}-x_{m, c}\right)=0
$$

We introduce an auxiliary variable $s_{m, c} \geq 0$ to relax and convert (54) into the posynomial inequalities as follows

$$
s_{m, c} \geq x_{m, c} y_{m}+1, \quad s_{m, c} \leq x_{m, c}^{2}+1
$$

In addition, the constraint $(47 \mathrm{~g})$ is a binary constraint which is intractable. To tackle it, we rewrite $(47 \mathrm{~g})$ in the equivalent form as

$$
0 \leq \psi_{m}^{n, c} \leq 1, \quad \sum_{m \in \mathcal{M}} \sum_{n \in \mathcal{N}} \psi_{m}^{n, c}-\left(\psi_{m}^{n, c}\right)^{2} \leq 0
$$

By utilizing the same method, to transform this constraint into the monotonic form as

$$
\sum_{m \in \mathcal{M}} \sum_{n \in \mathcal{N}}\left(\psi_{m}^{n, c}\right)^{2}+a \geq \mathcal{R}, \quad \sum_{m \in \mathcal{M}} \sum_{n \in \mathcal{N}} \psi_{m}^{n, c}+a \leq \mathcal{R}
$$

where $a$ is a slack variable and $\mathcal{R}$ is constant.

Hence, the final optimization problem (47) can be rewritten as

$$
\begin{aligned}
& \max _{\left(\mathbf{q}, \psi, t, \mathbf{s}, a, \mathcal{R}, Z_{1}\right)} Z_{1} \\
& \text { s.t. } \\
& g\left(q_{m, v}^{n, c}, \psi_{m}^{n, c}\right)+t \leq g(\mathbf{b}), \\
& h\left(q_{m, v}^{n, c}\right)+t \geq g(\mathbf{b}), \\
& 0 \leq t \leq g(\mathbf{b})-g(\mathbf{0}), \forall v \in \mathcal{V} \\
& \sum_{\forall v \in \mathcal{V}} \sum_{\forall n \in \mathcal{N}_{v}} \sum_{\forall m \in \mathcal{I}_{v}} q_{m, v}^{n, c} \leq P_{\max }^{c}, \forall c \in \mathcal{C} \\
& \sum_{\forall m \in \mathcal{M}} \psi_{m}^{n, c} \leq 1, \forall c \in \mathcal{C}, \forall n \in \mathcal{N}, \\
& s_{m, c} \geq x_{m, c} y_{m}+1, \quad s_{m, c} \leq x_{m, c}^{2}+1, \forall c \in \mathcal{C}, \forall m \in \mathcal{M} \\
& \psi_{m}^{n, c}=0, \forall m \in \mathcal{I}_{v} \text { and } n \in \mathcal{N} \backslash \mathcal{N}_{v}, \forall v \in \mathcal{V} \\
& 0 \leq \psi_{m}^{n, c} \leq 1, \quad \forall m \in \mathcal{M}, \forall n \in \mathcal{N} \\
& \sum_{m \in \mathcal{M}} \sum_{n \in \mathcal{N}}\left(\psi_{m}^{n, c}\right)^{2}+a \geq \mathcal{R}, \\
& \sum_{m \in \mathcal{M}} \sum_{n \in \mathcal{N}} \psi_{m}^{n, c}+a \leq \mathcal{R} .
\end{aligned}
$$


Consequently, the feasible set of (58) can be expressed as the intersection of two following sets:

$$
\begin{aligned}
& \mathcal{G}_{1}=\left\{\left\{q_{m . v}^{n, c}, \psi_{m}^{n, c}, t, s_{m, c}, a, Z_{1}\right\}: \mathbf{q} \preccurlyeq \mathbf{b}\right. \\
& (58 a),(58 d),(58 e),(58 h),(58 j)\} \\
& \mathcal{H}_{1}=\left\{\left\{q_{m . v}^{n, c}, \psi_{m}^{n, c}, t, s_{m, c}, a, Z_{1}\right\}: \mathbf{q} \succcurlyeq 0,(58 b),(58 i)\right\}
\end{aligned}
$$

Here, $\mathcal{G}_{1}$ is a normal set contained in box $[\mathbf{0}, \mathbf{b}] \times[0, g(\mathbf{b})-$ $g(0)]$, and $\mathcal{H}_{1}$ is a conormal set.

2) Rate-based Isolation Strategy: Assuming $Z_{2}=-\chi_{2}$, and apply the same procedures, rate-based isolation strategy can be expressed as the monotonic form

$$
\begin{aligned}
& \max _{\left(\mathbf{q}, \boldsymbol{\psi}, t, \mathbf{s}, a, w, \mathcal{R}, Z_{2}\right)} Z_{2} \\
& \text { s.t. } \\
& g\left(q_{m, v}^{n, c}, \psi_{m}^{n, c}\right)+t \leq g(\mathbf{b}), \\
& h\left(q_{m, v}^{n, c}\right)+t \geq g(\mathbf{b}), \\
& 0 \leq t \leq g(\mathbf{b})-g(\mathbf{0}), \forall v \in \mathcal{V} \\
& \sum_{\forall v \in \mathcal{V}} \sum_{\forall n \in \mathcal{N}_{v}} \sum_{\forall m \in \mathcal{I}_{v}} q_{m, v}^{n, c} \leq P_{\text {max }}^{c}, \forall c \in \mathcal{C} \\
& \sum_{\forall m \in \mathcal{M}} \psi_{m}^{n, c} \leq 1, \forall c \in \mathcal{C}, \forall n \in \mathcal{N}, \\
& s_{m, c} \geq x_{m, c} y_{m}+1, \quad s_{m, c} \leq x_{m, c}^{2}+1, \forall c \in \mathcal{C}, \forall m \in \mathcal{M} \\
& \psi_{m}^{n, c}=0, \forall m \in \mathcal{I}_{v} \text { and } n \in \mathcal{N} \backslash \mathcal{N}_{v}, \forall v \in \mathcal{V} \\
& 0 \leq \psi_{m}^{n, c} \leq 1, \quad \forall m \in \mathcal{M}, \forall n \in \mathcal{N} \\
& \sum_{m \in \mathcal{M}} \sum_{n \in \mathcal{N}}\left(\psi_{m}^{n, c}\right)^{2}+a \geq \mathcal{R}, \\
& \sum_{m \in \mathcal{M}} \sum_{n \in \mathcal{N}} \psi_{m}^{n, c}+a \leq \mathcal{R} \\
& g_{r}(\mathbf{q})+w \leq g_{r}\left(\mathbf{b}_{r}\right) \\
& h_{r}(\mathbf{q})+w \geq h_{r}\left(\mathbf{b}_{r}\right) \\
& 0 \leq w \leq g_{r}\left(\mathbf{b}_{r}\right)-g_{r}(\mathbf{0}) \\
& g_{r}\left(q_{m, v}^{n, c}\right)=R_{\min }^{v}+\sum_{\forall m \in \mathcal{I}_{v}} \sum_{\forall n \in \mathcal{N}_{v}} \sum_{\forall c \in \mathcal{C}} \\
& \times \ln \left(N_{0}+\sum_{\forall c^{\prime} \neq c \forall \forall v^{\prime} \in \mathcal{V}} q_{m, v^{\prime}}^{n, c^{\prime}} h_{m}^{n, c^{\prime}}+q_{m, n}^{n, c} h_{m}^{n, c}\right) \\
& h_{r}\left(q_{m, v}^{n, c}\right)=\sum_{\forall m \in \mathcal{I}_{v}} \sum_{\forall n \in \mathcal{N}_{v}} \sum_{\forall c \in \mathcal{C}} \ln \left(N_{0}+\sum_{\forall c^{\prime} \neq c} \sum_{\forall v^{\prime} \in \mathcal{V}} q_{m, v^{\prime}}^{n, c^{\prime}} h_{m}^{n, c^{\prime}}\right)
\end{aligned}
$$

Therefore, the feasible set of (61) can be expressed as

$$
\begin{aligned}
\mathcal{G}_{2}= & \left\{\left\{q_{m . v}^{n, c}, \psi_{m}^{n, c}, t, s_{m, c}, a, w, Z_{2}\right\}: \mathbf{q} \preccurlyeq \mathbf{b}\right. \\
& (61 a),(61 d),(61 e),(61 h),(61 j),(61 k),(61 l)\} \\
\mathcal{H}_{2}= & \left\{\left\{q_{m . v}^{n, c}, \psi_{m}^{n, c}, t, s_{m, c}, a, w, Z_{2}\right\}:\right. \\
& \mathbf{q} \succcurlyeq 0,(61 b),(61 i),(61 m)\}
\end{aligned}
$$

Here, $\mathcal{G}_{2}$ is a normal set contained in box $[\mathbf{0}, \mathbf{b}] \times[0, g(\mathbf{b}-$ $g(0))] \times\left[\mathbf{0}, \mathbf{b}_{r}\right] \times\left[0, g_{r}\left(\mathbf{b}_{r}\right)-g_{r}(0)\right]$, and $\mathcal{H}_{2}$ is a conormal set. Thus, those problems become monotonic optimization in canonial form. The optimal solutions can be found at the upper boundary of the feasible set by applying the ployblock outer approximation algorithm.

\section{Polyblock Outer Approximation Method}

For monotonic programming problems, [50] develops an outer approximation algorithm which is based on the approximation of compact normal sets by simpler normal sets, called polyblocks. The set of all proper vertices of a polyblock is called the proper vertex set. A polyblock is completely decided by its proper vertices. Further, a compact normal set can be approximated by a polyblock. It can be effortlessly demonstrated that the maximum of an increasing function over a polyblock is achieved at a proper vertex. By enumerating the vertices of the polyblock, the maximum value of the increasing function over the polyblock can be successfully obtained. Consequently, it is necessary to find an iterative method to generate a sequence of polyblocks of shrinking sizes such that

$$
\mathcal{P}_{1} \supset \mathcal{P}_{2} \ldots \supset \mathcal{P}_{k} \supset \ldots \mathcal{G} \cap \mathcal{H}
$$

such that

$$
\max \left\{f(x) \mid x \in \mathcal{P}_{k}\right\} \searrow \max \{f(x) \mid x \in \mathcal{G} \cap \mathcal{H}\}
$$

where $\searrow$ demonstrates that the polyblock outer approximation algorithm converges from above. since the maximum value of the increasing function over the feasible set $\mathcal{G} \cap \mathcal{H}$ is never greater than that over the polyblock $\mathcal{P}_{k}$ that encloses the feasible set. In addition, the size of the enclosing polyblock can be reduced via removing the vertices which are not in $\mathcal{H}$. Thus the obtained polyblock are still included in set of $\mathcal{G} \cap \mathcal{H}$. This procedure can be repeated until global maximum is found. The polyblock outer approximation algorithm mainly follows the following procedures.

- We need to find a polyblock $\mathcal{P}_{1}$ that encloses the feasible set $\mathcal{G} \cap \mathcal{H}$. Let $\mathcal{T}_{1}$ denote the proper vertex set of $\mathcal{P}_{1}$. Without loss of generality, the initial enclosing polyblock $\mathcal{P}_{1}$ can be simply constructed as $[\mathbf{0}, \mathbf{b}]$ and thus $\mathcal{T}_{1}=$ $\{\mathbf{b}\}$.

- We need to find the projection of vertex $\mathbf{z}_{k}$ onto the set $\mathcal{G}$, which is represented as $\pi_{\mathcal{G}}\left(\mathbf{z}_{k}\right)=\alpha \mathbf{z}_{k}, \alpha=[0,1]$, then the upper boundary point can be calculated by applying the bisection search. If the optimal vertex $\mathbf{z}_{k}$ over $\mathcal{T}_{k}$ happens to reside in feasible set, then it solves problem and its optimal solution $\mathbf{x}^{*}=\overline{\mathbf{x}}_{k}$. Otherwise, we need to construct a smaller $\mathcal{P}_{k+1} \subset \mathcal{P}_{k}$ that still contains $\mathcal{G}$ but excludes $\mathbf{z}_{k}$.

- We can repeat the procedure above until an optimal solution is found. In practice, we can terminate the algorithm when $\left|f\left(\mathbf{z}_{k}\right)-\Psi_{k}\right| \leq \epsilon$ is sufficiently small, where $\mathbf{z}_{k}$ denotes the optimal vertex that maximizes $f$ among all vertices of $\mathcal{P}_{k}$ and $\Psi_{k}$ is the current best value, and claim that $\mathbf{x}$ is optimal.

The detail of the polyblock outer approximation algorithm are summarized in Algorithm 2. 


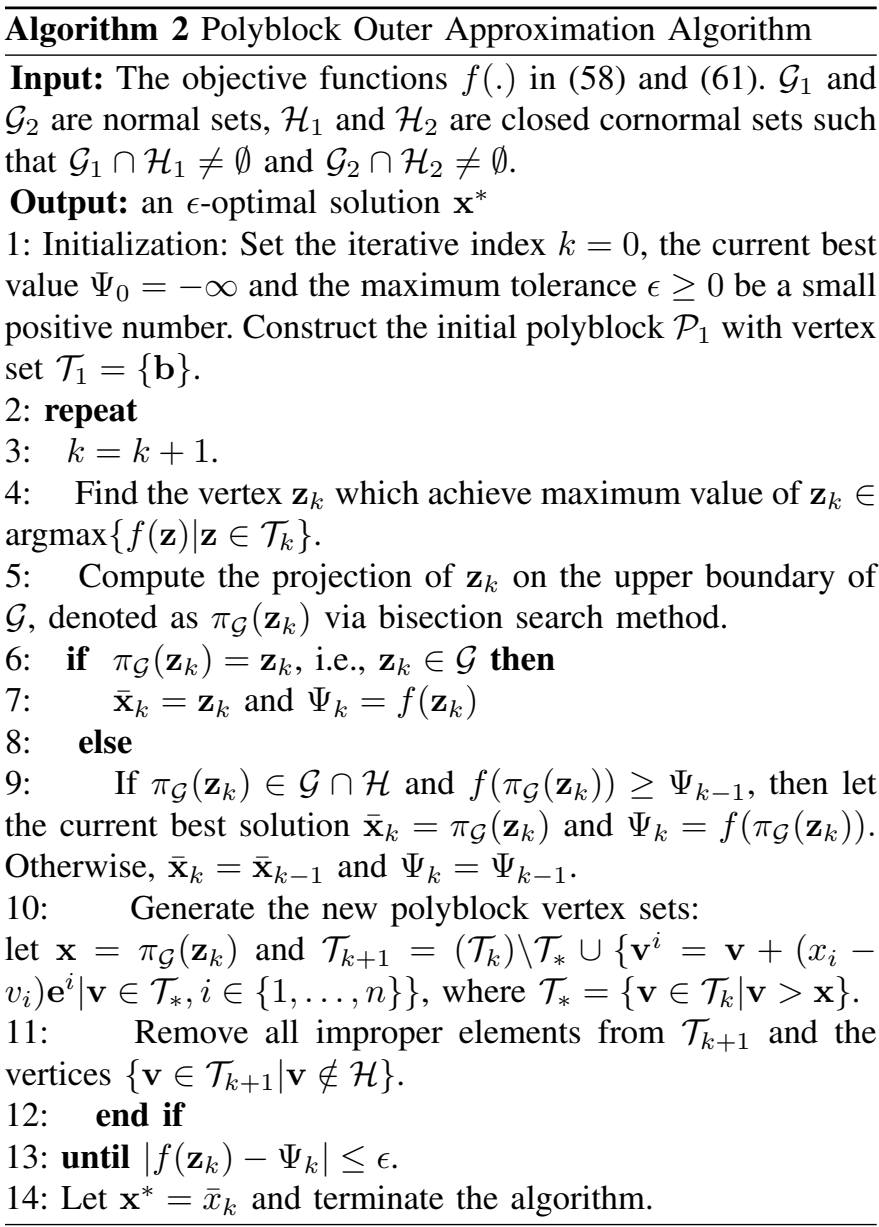

\section{Computational Complexity}

In this work, in order to find the global optimal solution, we investigated the monotonic optimization method by utilizing the polyblock outer approximation algorithm which includes four main step. First, find the best vertex whose projection belongs to the normal set. Next, the projection of the selected vertex on the normal set is obtained by using the bisection algorithm. Then, the improper vertexes that do not satisfy the co-normal constraints are deleted. Finally, the new vertex set is found based on the projection of the vertex on the upper boundary of the normal set. The algorithm continues until convergence and the best candidate vertex point is found with a simplified complexity order is $\mathcal{O}\left(L_{2}\left(L_{2} \times L_{1}+L_{3}\right)\right)$ where $L_{1}$ is the dimensions of the optimization problem. $L_{2}$ and $L_{3}$ are the numbers of iterations of the overall polyblock algorithm to converge in the bisection algorithm for the projection of each vertex, respectively.

\section{NUMERICAL RESULTS}

In this section, we present numerical results for a tradeoff analysis between the performance of MVNOs in OFDMAbased VWNs. In the presented numerical results, we assume that eight users $(M=8)$ are served by two cells $(C=2)$ where the maximum power level is $P_{\max }^{c}=10 \mathrm{~W}$ for each cell, and the minimum reserved rate for the rate-based strategy is fixed to $R_{\min }=0.1$ nats $/ \mathrm{s} / \mathrm{Hz}$, unless otherwise indicated.
We normalize the transmit power to the noise power assuming that $N_{0}=1$. Noting that in the following figures, in order to find the values of utopian objective points $U_{1}$ and $U_{2}$ used in (13) and (14), we separately solve different SOOPs. For example, in two MVNOs $(V=2)$, in the first SOOP, the total rate of the subscribers to the first $\mathrm{MVNO}$ is maximized while guaranteeing the total transmit power for each base station in each cell, whereas in the second SOOP, the rate of the users who subscribed to the second MVNO is maximized subject to a maximum transmit power constraint of each cell. Then, from these two SOOPs, Pareto optimal points are obtained as utopia points for $U_{1}$ and $U_{2}$. Moreover, we set $\kappa_{R}=1, \kappa_{P}=0.1$ and $\kappa_{B}=0.25$ to show the behaviour of the tradeoff between the sum profit of users in MVNOs.

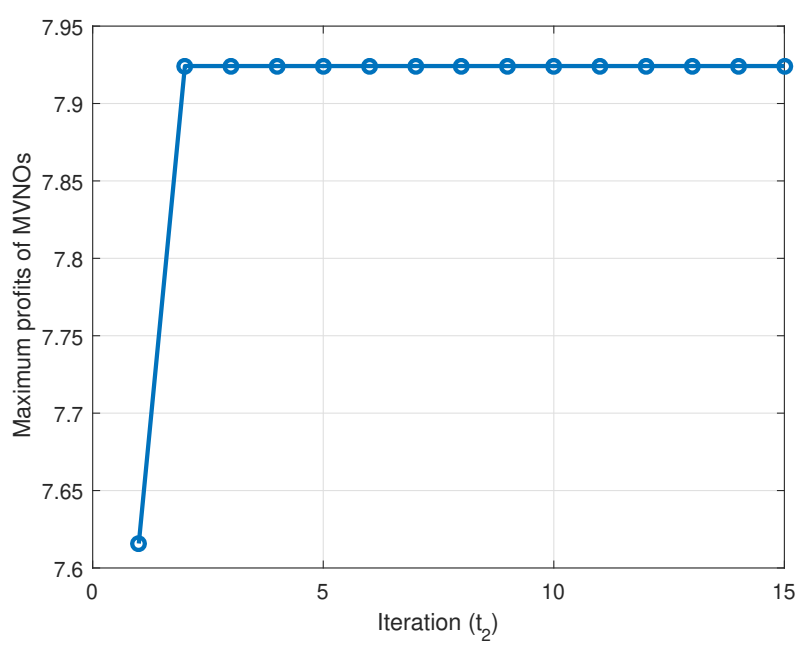

(a) DC Algorithm

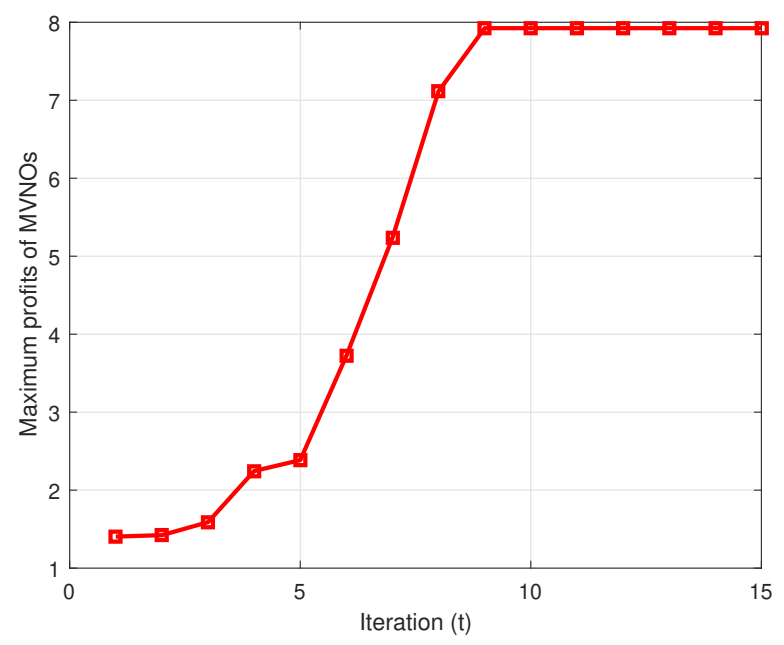

(b) Proposed Algorithm

Fig. 3. Convergence speed of Algorithm 1

In Fig. 3, we first examine the convergence speed of Algorithm 1, by plotting the number of iterations required for the DC algorithm and the outer loop in Algorithm 1 for the rate-based isolation strategy when $\alpha_{1}=0.5$. Clearly, based on Fig. 3a., DC algorithm converges quickly in only a few 
iterations. Therefore, we can conclude that DC algorithm has a fast convergence speed. In addition, the plot in Fig. 3b. reveals that the convergence of the proposed algorithm sequential subchannel assignment and power allocation occurs in less than 10 iterations.

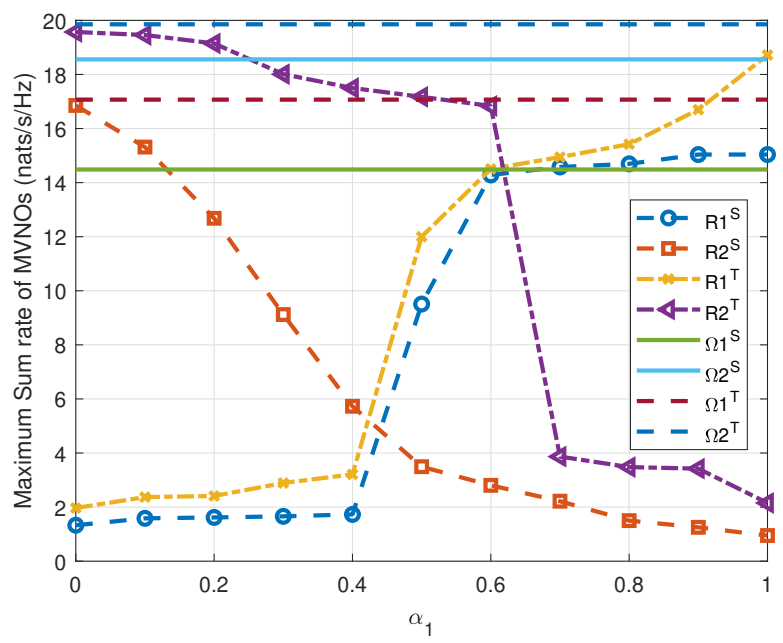

Fig. 4. Maximum sum rate of users in the first and second MVNO versus $\alpha_{1}$ for rate-based and resource-based isolation strategies

Fig. 4 shows the maximum sum rates of users in the first MVNO and the second MVNO versus $\alpha_{1}$ for rate-based and resource-based isolation strategies. From this figure, in both isolations, we observe that as the sum rate of users in the first MVNO increases, the sum rate of users in the second MVNO decreases which gives a tradeoff between the sum rates of users in these two MVNOs. It is evident that $\alpha_{1}$ as a weighting coefficient of MVNO 1 based on (7b) and (8b) can impact the achievable sum rate of MVNO 1. The utopia points of each MVNO for both isolation methods are shown in this figure as well. It can be observed the utopia points of each MVNO for the rated-based strategy is always higher than that for the resource-based one.

To compare the sum profit of users in both MVNOs in the case of the rate-based isolation strategy and the resource-based isolation strategy, Fig. 5 displays the maximum sum profit of users in both MVNOs versus $\alpha_{1}$ for both isolation strategies. In this figure, we observe that for all values of $\alpha_{1}$, the sum profit of users of MVNOs for rate-based isolation strategy is higher than the sum profit of users of MVNOs for the resource-based isolation strategy owing to the minimum rate guarantees. In addition, we include a monotonic optimization method in this figure to obtain the global optimal solutions to compare with the proposed suboptimal algorithm. It can be observed that the suboptimal GP method matches well with the monotonic optimization.

Since total power constraints of each cell is very important in our objective problems, we pay attention to the impact of power budget on the system performance. First, we focus on the SOOP problems, in Fig. 6. We investigate the utopia points versus $P_{\max }^{c}$ and we observe that utopia points are increasing functions of $P_{\max }^{c}$. Moreover, it is evident that utopia point

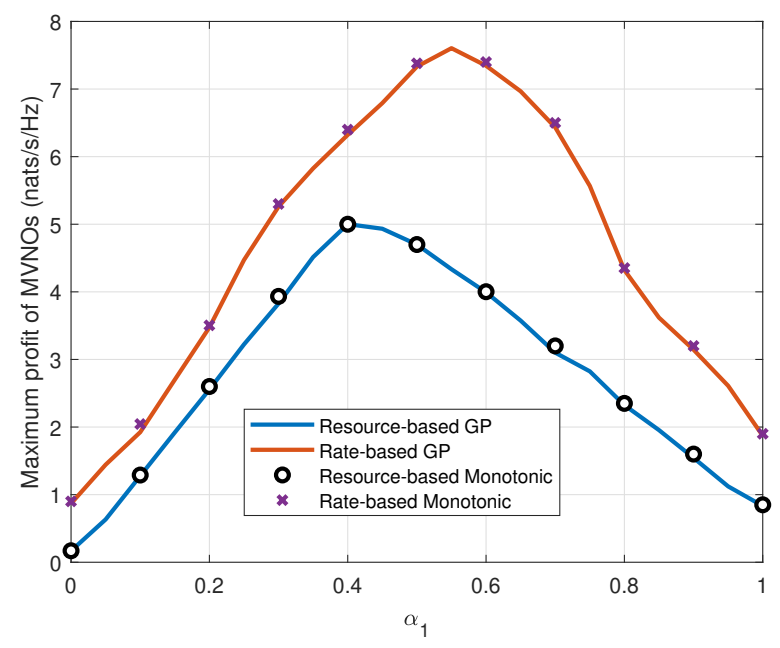

Fig. 5. Maximum sum profit of users in the both MVNOs versus $\alpha_{1}$ for rate-based and resource-based isolation strategies (Monotonic algorithm vs GP)

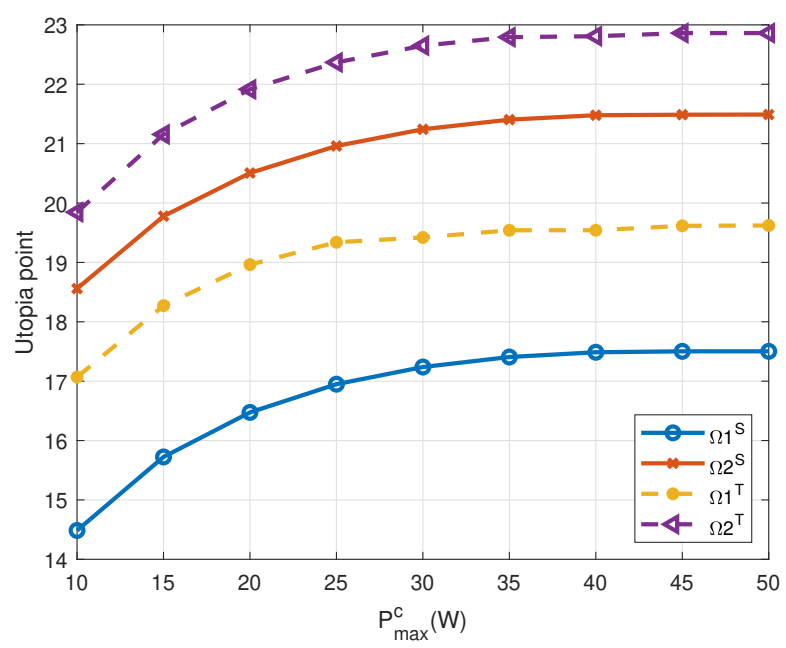

Fig. 6. Utopian points in the first and second MVNO versus $P_{\max }^{c}$ for ratebased and resource-based isolation strategies

of each MVNO for the resource-based isolation strategy is smaller than that for the rate-based isolation strategy. On the other hand, for the MOOP problem, we compare the sum profit behaviour between rate-based and resource-based isolation strategies in Fig. 7. Apparently, the sum profit increases with the growth of power value, and the sum profit for rate-based isolation strategies is always higher than that for resourcebased isolation strategies.

Fig. 8 shows the maximum profit gained by the first MVNO and the second MVNO versus $P_{\max }^{c}$ for rate-based and resource-based isolation strategies. From this figure, it can be seen that by varying $P_{\max }^{c}$ the profit for resourcebased decreases and the profit for rate-based increases. And we observe that for all values of $P_{\max }^{c}$ the sum profit of each MVNO for rate-based isolation strategy is higher than that of each MVNO for resource-based isolation strategy.

Fig. 9 demonstrates the effect of minimum reserved rate 


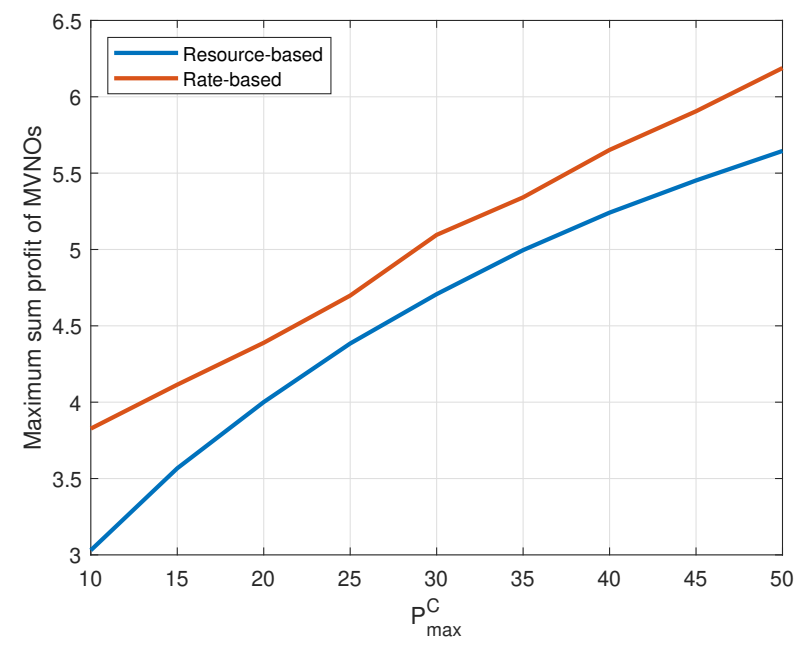

Fig. 7. Maximum sum profit of users in the both MVNOs versus $P_{\max }^{c}$ for rate-based and resource-based isolation strategies

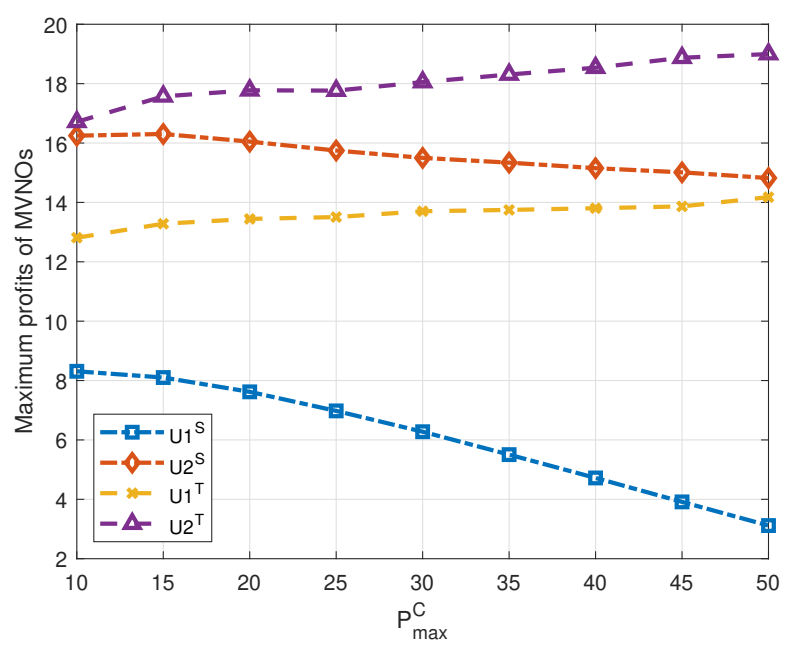

Fig. 8. Maximum sum profit of users in the first and second MVNO versus $P_{\max }^{c}$ for rate-based and resource-based isolation strategies

$R_{\min }$ on the maximum sum profit for rate-based isolation strategies. As we expected, as the minimum reserved rate increases, the maximum sum profit will increase, and it is suitable for different level of total power of each cell.

To investigate the case of three MVNOs $(V=3)$, Fig. 10 shows the relationship between maximum sum profit of users for each MVNO when $\alpha_{1}$ varies from 0 to $0.8, \alpha_{3}=0.2$ and $\alpha_{2}=1-\alpha_{1}-\alpha_{3}$ for both isolation strategies. In addition, the utopian point of each MVNO is $\Omega_{1}^{S}=11.7215, \Omega_{2}^{S}=$ $10.8994, \Omega_{3}^{S}=18.5588$ and $\Omega_{1}^{T}=13.0537, \Omega_{2}^{T}=21.2468$, $\Omega_{3}^{T}=19.8456$. From this figure, we observe that by varying $\alpha_{1}$, the profit of the first MVNO increases, the profit of the second MVNO decreases, and the profit of the third MVNO shows modest fluctuations. Moreover, for all values of $\alpha_{1}$, the maximum profit of users of each MVNO for the rate-based isolation strategy is higher than that for the resource-based isolation strategy owing to the minimum reserved rate.

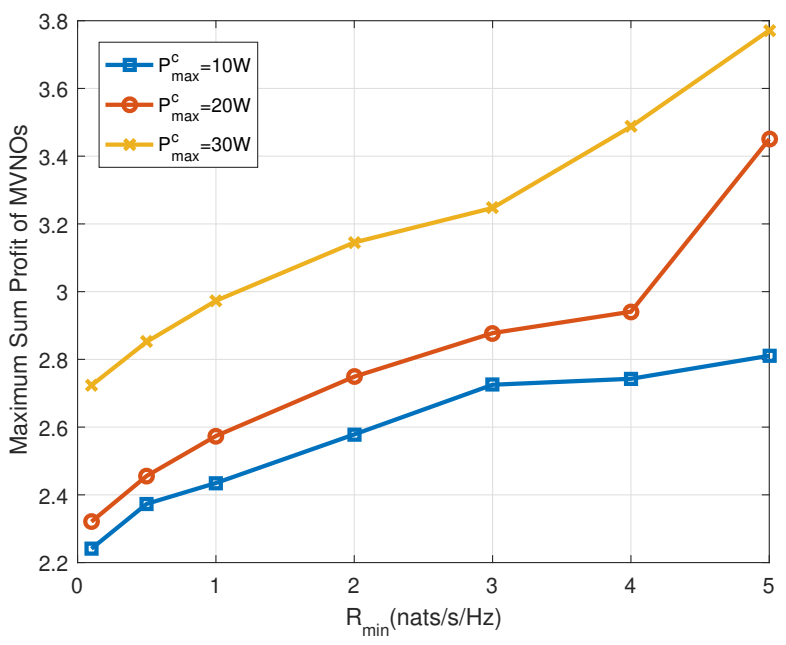

Fig. 9. Maximum sum profit of users in both MVNOs versus $R_{\min }$ for ratebased isolation strategies

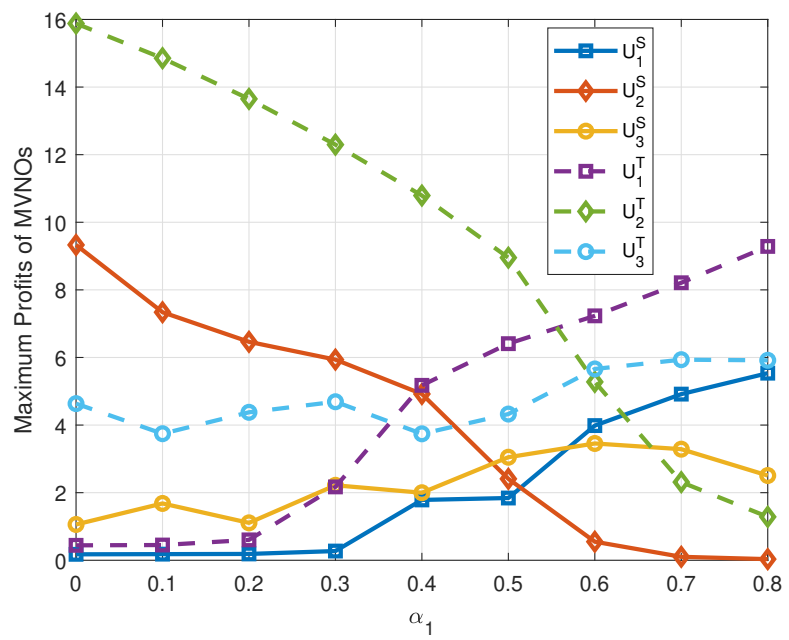

Fig. 10. Maximum sum profit of users in the first, second and third MVNO versus $\alpha_{1}$ for rate-based and resource-based isolation strategies

\section{CONCLUSION}

In this paper, we considered a downlink multi-cell OFDMAbased VWN. First, we presented an optimal power allocation strategy and subchannel assignment that gives a novel performance tradeoff between profit of MVNOs. To investigate this tradeoff between MVNOs, we formulated a MOOP that were solved by using a weighted Tchebycheff method for resourcebased and rate-based isolation strategies. Iterative approaches alternatively assigned subchannels to users in both MVNOs and allocated powers to MVNOs. Illustrative results show a fundamental performance tradeoff between these MVNOs, which leads to achieving the most suitable allocated resources by MVNO, regarding different importance levels of MVNOs. The monotonic optimization method was applied for solving the proposed optimization problem to obtain the global optimal solutions. The corresponding results show that the GP method matches well with the monotonic optimization. 


\section{REFERENCES}

[1] P. Cerwall, P. Jonsson, R. Möller, S. Bävertoft, S. Carson, and I. Godor, "Ericsson mobility report," On the Pulse of the Networked Society. Hg. v. Ericsson, 2015.

[2] C. Liang and F. R. Yu, "Wireless network virtualization: A survey, some research issues and challenges," IEEE Communications Surveys \& Tutorials, vol. 17, no. 1, pp. 358-380, 2014.

[3] J. van de Belt, H. Ahmadi, and L. E. Doyle, "Defining and surveying wireless link virtualization and wireless network virtualization," IEEE Communications Surveys Tutorials, vol. 19, no. 3, pp. 1603-1627, 2017.

[4] M. M. Hasan, H. Amarasinghe, and A. Karmouch, "Network virtualization: Dealing with multiple infrastructure providers," in IEEE International Conference on Communications (ICC), 2012, pp. 58905895.

[5] A. Xiao, Y. Liu, Y. Li, F. Qian, Z. Li, S. Bai, Y. Liu, T. Xu, and X. Xin, "An in-depth study of commercial MVNO: Measurement and optimization," in Proceedings of the 17th Annual International Conference on Mobile Systems, Applications, and Services, 2019, pp. 457-468.

[6] F. Zarinni, A. Chakraborty, V. Sekar, S. R. Das, and P. Gill, "A first look at performance in mobile virtual network operators," in Proceedings of the conference on internet measurement conference, 2014, pp. 165-172.

[7] Y. Li, J. Zheng, Z. Li, Y. Liu, F. Qian, S. Bai, Y. Liu, and X. Xin, "Understanding the ecosystem and addressing the fundamental concerns of commercial MVNO," IEEE/ACM Transactions on Networking, vol. 28, no. 3, pp. 1364-1377, 2020.

[8] P. Schmitt, M. Vigil, and E. Belding, "A study of MVNO data paths and performance," in International Conference on Passive and Active Network Measurement. Springer, 2016, pp. 83-94.

[9] Y. K. Tun, N. H. Tran, D. T. Ngo, S. R. Pandey, Z. Han, and C. S. Hong, "Wireless network slicing: Generalized kelly mechanism-based resource allocation," IEEE Journal on Selected Areas in Communications, vol. 37, no. 8, pp. 1794-1807, 2019.

[10] L. Li, N. Deng, W. Ren, B. Kou, W. Zhou, and S. Yu, "Multi-service resource allocation in future network with wireless virtualization," IEEE Access, vol. 6, pp. 53854-53 868, 2018.

[11] R. Kokku, R. Mahindra, H. Zhang, and S. Rangarajan, "NVS: A substrate for virtualizing wireless resources in cellular networks,' IEEE/ACM transactions on networking, vol. 20, no. 5, pp. 1333-1346, 2011.

[12] M. Balon and B. Liau, "Mobile virtual network operator," in 15th International Telecommunications Network Strategy and Planning Symposium (NETWORKS), 2012, pp. 1-6.

[13] P. Kalmus and L. Wiethaus, "On the competitive effects of mobile virtual network operators," Telecommunications Policy, vol. 34, no. 5-6, pp. 262-269, 2010.

[14] Y. Coulibaly, G. Kaddoum, and M. F. A. Ahmed, "Mobile virtual network operator strategy for migration towards 4G," in International Conference on Information and Communication Technology Research (ICTRC), 2015, pp. 266-269.

[15] M. Richart, J. Baliosian, J. Serrat, and J.-L. Gorricho, "Resource slicing in virtual wireless networks: A survey," IEEE Transactions on Network and Service Management, vol. 13, no. 3, pp. 462-476, 2016.

[16] X. Costa-Pérez, J. Swetina, T. Guo, R. Mahindra, and S. Rangarajan, "Radio access network virtualization for future mobile carrier networks," IEEE Communications Magazine, vol. 51, no. 7, pp. 27-35, 2013.

[17] S. Parsaeefard, R. Dawadi, M. Derakhshani, and T. Le-Ngoc, "Joint userassociation and resource-allocation in virtualized wireless networks," IEEE Access, vol. 4, pp. 2738-2750, 2016.

[18] T. LeAnh, N. H. Tran, D. T. Ngo, and C. S. Hong, "Resource allocation for virtualized wireless networks with backhaul constraints," IEEE Communications Letters, vol. 21, no. 1, pp. 148-151, 2016.

[19] S. Parsaeefard, V. Jumba, M. Derakhshani, and T. Le-Ngoc, "Joint resource provisioning and admission control in wireless virtualized networks," in IEEE Wireless Communications and Networking Conference (WCNC). IEEE, 2015, pp. 2020-2025.

[20] D. B. Rawat, B. S. Alsulami, and V. Chaudhary, "On the wireless virtualization with QoE constraints," Transactions on Emerging Telecommunications Technologies, vol. 30, no. 3, p. e3573, 2019.

[21] Z. Li and A. Zhong, "Resource allocation in wireless powered virtualized sensor networks," IEEE Access, vol. 8, pp. 40327-40336, 2020.

[22] L. P. Qian, Y. Wu, H. Zhou, and X. Shen, "Joint uplink base station association and power control for small-cell networks with nonorthogonal multiple access," IEEE Transactions on Wireless Communications, vol. 16, no. 9, pp. 5567-5582, 2017.
[23] P. Luoto, P. Pirinen, M. Bennis, S. Samarakoon, S. Scott, and M. Latvaaho, "Co-primary multi-operator resource sharing for small cell networks," IEEE Transactions on Wireless Communications, vol. 14, no. 6 , pp. 3120-3130, 2015.

[24] T. M. Ho, N. H. Tran, S. M. Ahsan Kazmi, and C. S. Hong, "Dynamic pricing for resource allocation in wireless network virtualization: A stackelberg game approach," in International Conference on Information Networking (ICOIN), 2017, pp. 429-434.

[25] M. H. Lotfi and S. Sarkar, "The economics of competition and cooperation between MNOs and MVNOs," in 51st Annual Conference on Information Sciences and Systems (CISS), 2017, pp. 1-6.

[26] C. Wu, R. Wang, P. Wang, Y. Cao, L. Liu, K. Zhu, and B. Chen, "On the profit maximization of spectrum investment under uncertainties in cognitive radio networks," in IEEE International Conference on Communications (ICC), 2018, pp. 1-6.

[27] C. Li, J. Li, Y. Li, and Z. Han, "Pricing game with complete or incomplete information about spectrum inventories for mobile virtual network operators," IEEE Transactions on Vehicular Technology, vol. 68, no. 11 , pp. $11118-11131,2019$.

[28] F. Sun, F. Hou, H. Zhou, B. Liu, J. Chen, and L. Gui, "Equilibriums in the mobile-virtual-network-operator-oriented data offloading," IEEE Transactions on Vehicular Technology, vol. 67, no. 2, pp. 1622-1634, 2018.

[29] J. Ye and Y.-J. Zhang, "Pricing-based resource allocation in virtualized cloud radio access networks," IEEE Transactions on Vehicular Technology, vol. 68, no. 7, pp. 7096-7107, 2019.

[30] N. N. Sapavath and D. B. Rawat, "Wireless virtualization architecture: Wireless networking for internet of things," IEEE Internet of Things Journal, vol. 7, no. 7, pp. 5946-5953, 2020.

[31] R. T. Marler and J. S. Arora, "Survey of multi-objective optimization methods for engineering," Structural and multidisciplinary optimization, vol. 26, no. 6, pp. 369-395, 2004.

[32] J. Rubio, A. Pascual-Iserte, D. P. Palomar, and A. Goldsmith, "Joint optimization of power and data transfer in multiuser MIMO systems," IEEE Transactions on Signal Processing, vol. 65, no. 1, pp. 212-227, 2016.

[33] P. Luong, F. Gagnon, C. Despins, and L.-N. Tran, "Optimal joint remote radio head selection and beamforming design for limited fronthaul CRAN," IEEE Transactions on Signal Processing, vol. 65, no. 21, pp. 5605-5620, 2017.

[34] M. R. Mili, L. Musavian, and D. W. K. Ng, "Rate-power-interference optimization in underlay OFDMA CRNs with imperfect CSI," IEEE Communications Letters, vol. 21, no. 7, pp. 1657-1660, 2017.

[35] H. Hu, Y.-C. Liang, H. Zhang, and B.-H. Soong, "Cognitive radio with self-power recycling," IEEE Transactions on Vehicular Technology, vol. 66, no. 7, pp. 6201-6214, 2016.

[36] O. Aydin, E. A. Jorswieck, D. Aziz, and A. Zappone, "Energy-spectral efficiency tradeoffs in $5 \mathrm{G}$ multi-operator networks with heterogeneous constraints," IEEE Transactions on Wireless Communications, vol. 16, no. 9, pp. 5869-5881, 2017.

[37] C. C. Coskun and E. Ayanoglu, "Energy-spectral efficiency tradeoff for heterogeneous networks with QoS constraints," in IEEE International Conference on Communications (ICC). IEEE, 2017, pp. 1-7.

[38] D. W. K. Ng, E. S. Lo, and R. Schober, "Multi objective resource allocation for secure communication in cognitive radio networks with wireless information and power transfer," IEEE Transactions on Vehicular Technology, vol. 65, no. 5, pp. 3166-3184, 2016.

[39] Y. Sun, D. W. K. Ng, J. Zhu, and R. Schober, "Multi-objective optimization for robust power efficient and secure full-duplex wireless communication systems," IEEE Transactions on Wireless Communications, vol. 15, no. 8, pp. 5511-5526, 2016.

[40] Z. Li, S. Gong, C. Xing, Z. Fei, and X. Yan, "Multi-objective optimization for distributed MIMO networks," IEEE Transactions on Communications, vol. 65, no. 10, pp. 4247-4259, 2017.

[41] G. Yu, Y. Jiang, L. Xu, and G. Y. Li, "Multi-objective energy-efficient resource allocation for multi-RAT heterogeneous networks," IEEE Journal on Selected Areas in Communications, vol. 33, no. 10, pp. 2118-2127, 2015.

[42] M. R. Mili, F. Mokhtari, and F. Ashtiani, "Improving tradeoff among downlink rates of service providers in a VWN by using NOMA," IEEE Communications Letters, vol. 23, no. 1, pp. 156-159, 2018.

[43] K. Miettinen, Nonlinear multiobjective optimization. Springer Science \& Business Media, 2012, vol. 12.

[44] M. Avriel and A. Williams, "Complementary geometric programming," SIAM Journal on Applied Mathematics, vol. 19, no. 1, pp. 125-141, 1970 . 
[45] N. Vucic, S. Shi, and M. Schubert, "DC programming approach for resource allocation in wireless networks," in 8th International Symposium on Modeling and Optimization in Mobile, Ad Hoc, and Wireless Networks, May 2010, pp. 380-386.

[46] S. Boyd, S. P. Boyd, and L. Vandenberghe, Convex optimization. Cambridge university press, 2004.

[47] M. Chiang, C. W. Tan, D. P. Palomar, D. O'neill, and D. Julian, "Power control by geometric programming," IEEE Transactions on Wireless Communications, vol. 6, no. 7, pp. 2640-2651, 2007.

[48] B. R. Marks and G. P. Wright, "A general inner approximation algorithm for nonconvex mathematical programs," Operations research, vol. 26 , no. 4, pp. 681-683, 1978

[49] D. P. Bertsekas, "Nonlinear programming," Journal of the Operational Research Society, vol. 48, no. 3, pp. 334-334, 1997.

[50] H. Tuy, "Monotonic optimization: Problems and solution approaches," SIAM Journal on Optimization, vol. 11, no. 2, pp. 464-494, 2000.

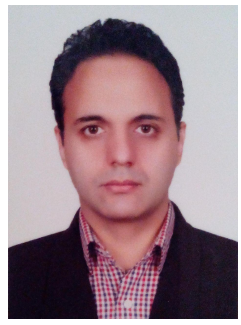

Mohammad Robat Mili received the Ph.D. degree in electrical and electronic engineering from the University of Manchester, U.K., in 2012. He held postdoctoral research positions at the Department of Telecommunications and Information Processing, Ghent University, Belgium and the Department of Electrical Engineering, Sharif University of Technology, Iran. His main research interests are in the area of design and analysis of wireless communication networks with particular focus on $5 \mathrm{G}$ and $6 \mathrm{G}$ celoptimization theory, game theory, and machine learning.

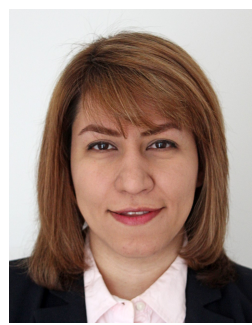

Mahsa Derakhshani (Senior Member, IEEE) received the Ph.D. degree in electrical engineering degree from McGill University, Montréal, Canada, in 2013. She was an Honorary NSERC Postdoctoral Fellow with the Department of Electrical and Electronic Engineering, Imperial College London, from 2015 to 2016, a Post-Doctoral Research Fellow with the Department of Electrical and Computer Engineering, University of Toronto, Toronto, Canada, and a Research Assistant with the Department of Electrical and Computer Engineering, McGill University, from 2013 to 2015. She is currently a Senior Lecturer in digital communications with the Wolfson School of Mechanical, Electrical and Manufacturing Engineering, Loughborough University, U.K. She received several awards and fellowships, including Royal Academy of Engineering/The Leverhulme Trust Research Fellowship (2020-21), the Natural Sciences and Engineering Research Council of Canada (NSERC) Postdoctoral Fellowships (2015-17), the Fonds de Recherche du Québec-Nature et Technologies (FRQNT) Postdoctoral Fellowship (2013-2015), and the John Bonsall Porter Prize (2009-10), the McGill Engineering Doctoral Award (2008-11). Her current research interests include machine learning and optimization for communications, ultra-reliable low-latency communications, software defined wireless networking, mobile edge caching. She serves as the Editor for IEEE Wireless Communications Letters, IEEE IoT Magazine, and IET Signal Processing Journal.

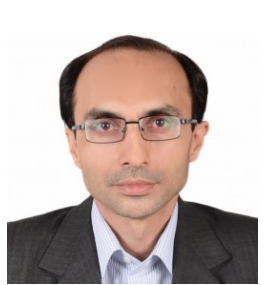

Farid Ashtiani received his Ph.D. degree from Sharif University of Technology, Tehran, Iran, in 2003, in Electrical Engineering. From 1995 to 1999 , he was partly working at the Power Research Center (P.R.C.) and Niroo Research Institute (N.R.I.) of Iran. From 1999 to 2001, he was a member of research staff with the Advanced Communication Science Research Laboratory, Iran Telecommunication Research Center (I.T.R.C.), Tehran, Iran. Since 2003, he has been with the Department of Electrical Engineering, Sharif University of Technology, where he is currently an Associate Professor and a technical member of Advanced Communications Research Institute (ACRI). His research interests include queueing theory, modeling, analysis, and design of different types of wireless networks, and mobility modeling.

Shenhong Li received M.Sc. degree in mobile

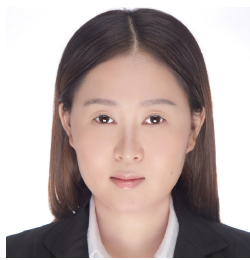
communications and Ph.D degree in electronic and electrical engineering from the Loughborough University, UK in 2014 and 2020, respectively. She was an Intern at Nokia Bell Labs, France. She is currently a Post-Doctoral Researcher with the School of Information Science and Technology, Nantong University, China. Her research interests include non-orthogonal multiple access, resource allocation in wireless networks and optimization problems.

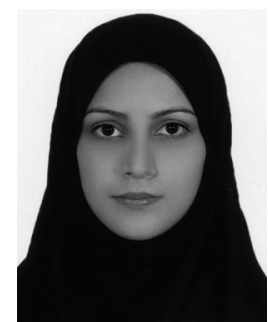

Fatemeh Mokhtari received the B.Sc., M.Sc. and $\mathrm{Ph} . \mathrm{D}$. degrees in electrical engineering from the Sharif University of Technology, Tehran, Iran, in 2007, 2010 and 2020 respectively. Her research interests include non-orthogonal multiple access and $5 \mathrm{G}$ wireless networks.

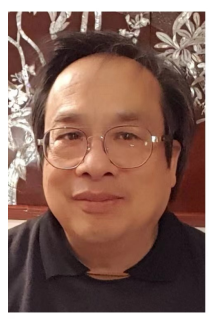

Tho Le-Ngoc (Life Fellow, IEEE) received the B.Eng. degree in electrical engineering in 1976, the M.Eng. degree in microprocessor applications from McGill University, Montreal, in 1978, and the Ph.D. degree in digital communications from the University of Ottawa, Canada, in 1983. From 1977 to 1982, he was with Spar Aerospace Ltd., Sainte-Anne-de-Bellevue, QC, Canada, involved in the development and design of satellite communications systems. From 1982 to 1985, he was with SRTelecom, Inc., Saint Laurent, QC, Canada, where he developed the new point-to-multipoint DA-TDMA/TDM Subscriber Radio System SR500. From 1985 to 2000, he was a Professor with the Department of Electrical and Computer Engineering, Concordia University, Montreal. Since 2000, he has been with the Department of Electrical and Computer Engineering, McGill University. His research interest includes broadband digital communications. He was a recipient of the 2004 Canadian Award in Telecommunications Research and the IEEE Canada Fessenden Award in 2005. He is a Distinguished James McGill Professor, and a Fellow of the Engineering Institute of Canada, the Canadian Academy of Engineering, and the Royal Society of Canada. 